\title{
High-resolution spectro-polarimetry of a flaring sunspot penumbra ${ }^{\star}$
}

\author{
J. Hirzberger ${ }^{1}$, T. Riethmüller ${ }^{1}$, A. Lagg $^{1}$, S. K. Solanki ${ }^{1,2}$, and P. Kobel ${ }^{1}$ \\ 1 Max-Planck-Institut für Sonnensystemforschung, Katlenburg-Lindau, Germany \\ e-mail: hirzberger@mps.mpg.de \\ 2 School of Space Research, Kyung Hee University, Yongin, Gyeonggi 446-71, Korea
}

Received 13 March 2009 / Accepted 4 June 2009

\section{ABSTRACT}

\begin{abstract}
We present simultaneous photospheric and chromospheric observations of the trailing sunspot in NOAA 10904 during a weak flare eruption (GOES magnitude B7.8), obtained with the Swedish Solar Telescope (SST) in La Palma, Canary Islands. High-resolution Ca II $H$ images show a typical two-ribbon structure that has been hitherto only known for larger flares, and the flare appears in a confined region that is discernible by a bright border. The underlying photosphere shows a disturbed penumbral structure with intersecting branches of penumbral filaments. High-resolution Doppler- and vector-magnetograms exhibit oppositely directed Evershed flows and magnetic field vectors in the individual penumbral branches, resulting in several regions of magnetic azimuth discontinuity and several islands where the vertical magnetic field is reversed. The discontinuity regions are co-spatial with the locations of the onset of the flare ribbons. From the results, we conclude that the confined flare region is detached from the global magnetic field structure by a separatrix marked by the bright border visible in $\mathrm{Ca}$ II $H$. We further conclude that the islands of reversed vertical field appear because of flux emergence and that the strong magnetic shear appearing in the regions of magnetic azimuth discontinuity triggers the
\end{abstract} flare.

Key words. Sun: flares - Sun: sunspots - Sun: magnetic fields - techniques: polarimetric - techniques: high angular resolution

\section{Introduction}

Solar flares are most likely caused by a sudden release of magnetic energy due to plasma instabilities or magnetic reconnection. The dynamics of large X-class flares has been intensely studied in the past decades (see e.g. Li et al. 2000a,b; Wang 2006; Su et al. 2007; Benz 2008, and references therein). The results are still inconclusive for the trigger, although magnetic shear, defined as the difference between the magnetic azimuths of the observed field and a potential field, around the magnetic neutral lines (Hagyard et al. 1984) and fast magnetic flux emergence (Schmieder et al. 1994) may play an important role in their formation. These studies were mainly based on low to moderate resolution observations.

The structure of smaller flares is much less clear since an in-depth study requires higher resolution observations, particularly, magnetograms. The importance of studying small flares is given by their much higher frequency of occurrence, which scales with a power law of index -1.8 (Lin et al. 1984) on the released energy. Thus, they may provide clues on the mechanisms causing micro- and nanoflares since the thermal events found in the corona scale with the same power law (Wang et al. 2006), but see Pauluhn \& Solanki (2007). There are many other reasons that the study of smaller flares is relevant for understanding the coronal heating (see Aschwanden et al. 2007, and the review by Krucker 2002).

The present study is based on the fortunate coincidence, that during a multi-wavelength observation run of a mature sunspot, a small flare erupted above its penumbra. We present time series of

\footnotetext{
^ Movies are only available in electronic form at http://www . aanda.org
}

high-resolution filtergrams, together with vector magnetograms of hitherto unprecedented spatial resolution. The photospheric magnetic field and flow structure is analysed and related to the scenario for flaring in the lower chromosphere.

\section{Observations and data reduction}

\subsection{Observations}

On August 13, 2006 multi-wavelength observations of the trailing sunspot of the active region NOAA 10904 (see Figs. 1 and 23) were carried out at the Swedish Solar Telescope (SST) in La Palma, Canary Islands (Scharmer et al. 2003). The centre of the field of view was located at solar disk coordinates $\left(x=-556^{\prime \prime}, y=-254^{\prime \prime}\right)$, which corresponds to a heliocentric angle of $\theta=40.15^{\circ}(\mu=0.76)$.

Close to the $F / 47$ focus of the SST, the sunlight was divided into a blue and a red channel by using a dichroic mirror plate. The instrumental setup for the blue beam was arranged with four Kodak Megaplus 1.6 CCD cameras and various interference filters. To obtain information about the lower photosphere, one of the cameras was fed light at the bandhead of the $\mathrm{CH}$ molecule at $\lambda=4305 \AA \pm 6.5 \AA$ (G-Band), and two cameras were fed with the light passing an $11 \AA$ interference filter with a central wavelength of $4363 \AA$ (G-continuum). One of these cameras was set slightly out of focus to allow subsequent image reconstruction by means of phase-diversity wavefront sensing. (The corresponding data were not used for the present study.) Information on the upper photosphere and the lower chromosphere was obtained using a tiltable narrow-band filter $(F W H M=1.1 \AA)$ in front of the 4 th camera. By tilting this filter, the calcium Ca II $H 3968.5 \AA$ line 


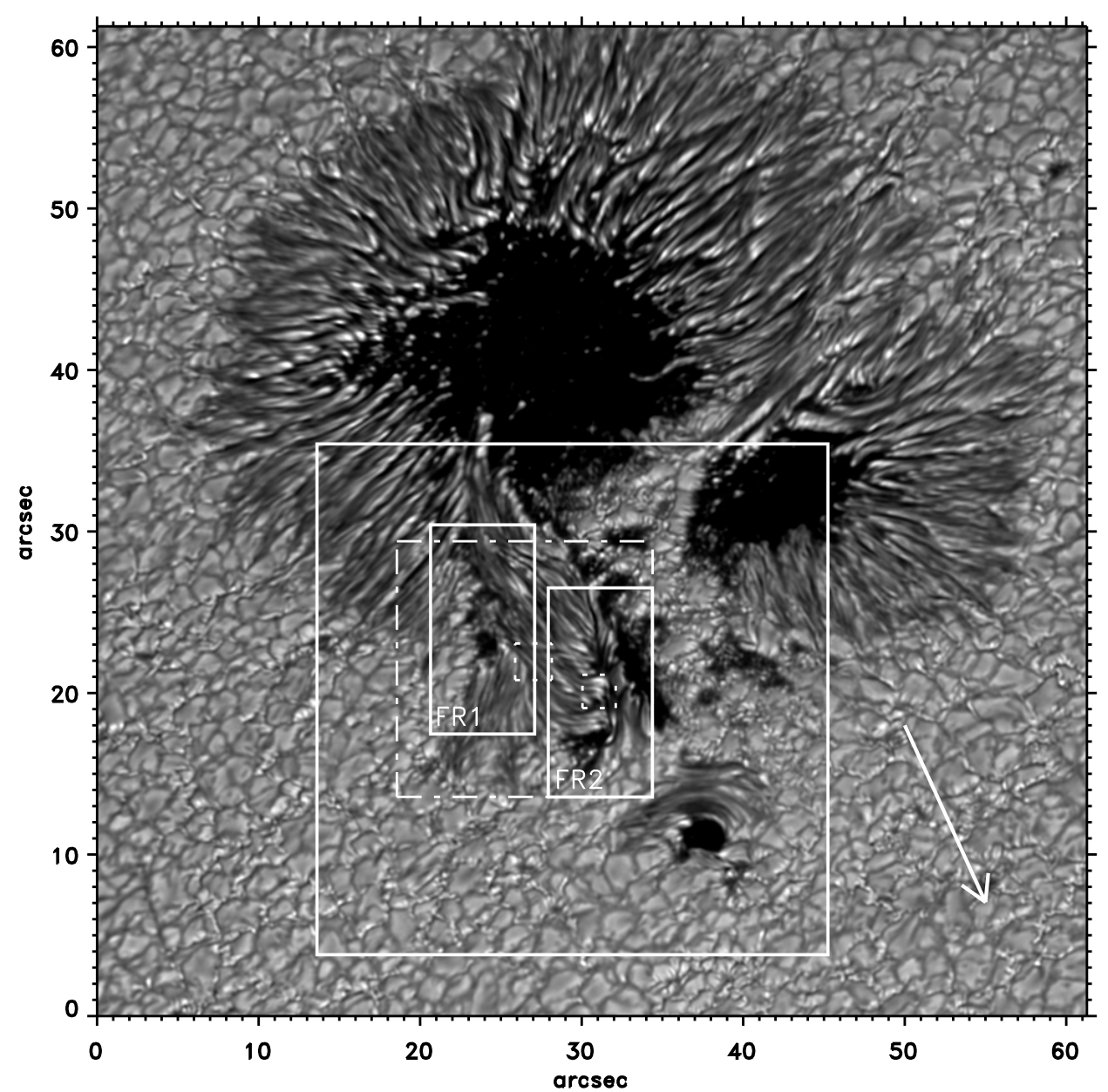

Fig. 1. Continuum image (broad band at $6302 \AA$ ) of the trailing sunspot in NOAA 10904 on August 13, 2006, 8:44:31 UT. The solid square marks the disturbed penumbra region as displayed in Figs. 2, 3, 5-7, 9, 10, and 13. The two solid rectangular boxes mark the two flare regions shown in Figs. 14-16 (right) and Figs. 17-19 (left). The dash-dotted square marks the subregions displayed in Fig. 12 and the small dotted squares show the subfields displayed in Figs. 8 (left) and 21 (right). The white arrow points towards disk centre.

was recorded at two wavelength points, once in the line centre and once in the blue wing, approximately $0.6 \AA$ out of the line centre. The two wavelength positions were alternated after recording four frames at a time. The exposure time was set to $13 \mathrm{~ms}$ for all four cameras in the blue beam, and the achieved frame rate was between 3 and 4 frames per second (G-band, G-continuum). Scanning the $\mathrm{Ca}$ II $H$-line needed slightly less than $6 \mathrm{~s}$. In the blue beam, the size of one pixel corresponds to $0 . " 041$.

The red channel was equipped with three Sarnoff CAM1M100 cameras, which were operated at a frame rate of $36 \mathrm{~s}^{-1}$. The Solar Optical Universal Polarimeter (SOUP, see Title \& Rosenberg 1981) filter was used to scan the iron Fe I $6302.5 \AA$ line at 6 wavelength positions $\left(\lambda_{0}-\lambda=\right.$ $[-250,-150,-75,0,75,150] \mathrm{m} \AA)$. Full Stokes polarimetry was performed by modulating the beam with two liquid crystal variable retarder (LCVRs), which allows the full magnetic field vector to be measured at each pixel of the field of view. In addition, two cameras were used to obtain continuum light (broad band) information at $\lambda=6302 \AA$. Again, one of these two cameras was slightly out of focus and the corresponding data were not used for the present study. The exposure time in the red beam was set to $4.5 \mathrm{~ms}$ by using a rotating shutter that rotated with a speed of approximately $36 \mathrm{~s}^{-1}$ and opens the beam during one sixth of each rotation. The remaining $23 \mathrm{~ms}$ of each shutter rotation were needed for detector read out and storage of the frames. The three cameras were operated in a master-slave mode so that the exposures were strictly simultaneous. At each wavelength position, 500 frames were recorded at any one time. The four polarisation states were alternated from one frame to the next. Tuning the SOUP instrument required several seconds for each wavelength position so that a full scan across the iron lines needed about $123 \mathrm{~s}$. The pixel size in the red beam corresponds to $0 . " 065$.

The seeing conditions were excellent during a period of more than four hours, so that the SST adaptive optics system was able to lock on the observed region almost uninterruptedly from the start of observations at 8:04:55 UT. Unfortunately, the SOUP filter system was suffering from several software problems, so that scanning of the iron line was started only at 8:28:51 UT and several interruptions had to be accepted.

\subsection{Image reconstruction}

The G-band and the G-continuum data were bundled into packages of 60 frames. Residual influences of the Earth's atmosphere were restored by reconstructing each of these packages with the help of speckle interferometric techniques (Weigelt 1977; Pehlemann \& von der Lühe 1989; de Boer 1996). To 


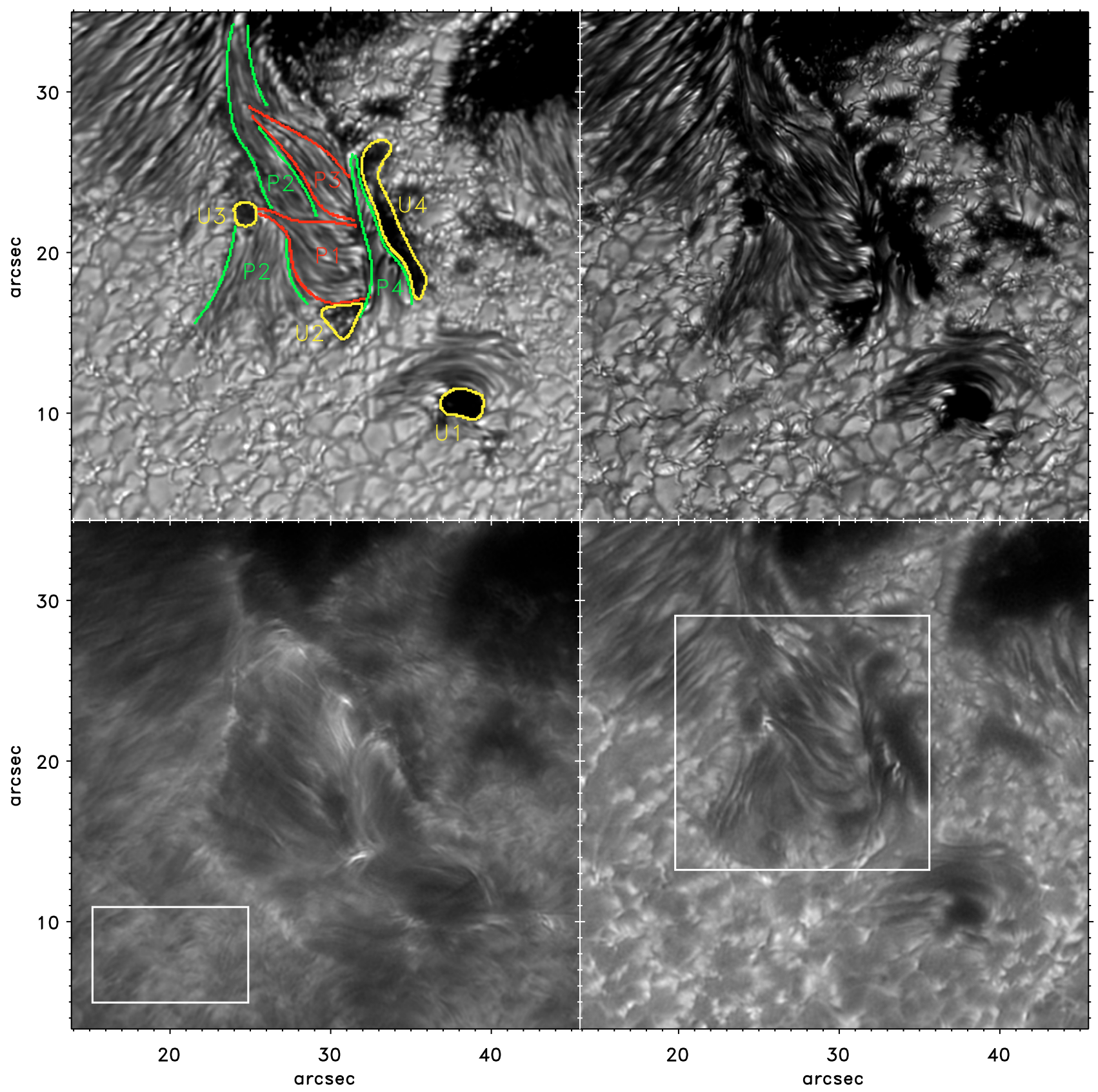

Fig. 2. Region of interest as observed on August 13, 2006 at the onset of the flare. Upper left: red continuum at 8:45:11 UT, upper right: G-continuum, lower left: Ca II $H$ line centre, lower right: Ca II $H-0.6 \AA$. The images from the blue channel were obtained at 8:44:59 UT. In the red continuum, image the individual branches of the disturbed penumbra region and four small umbrae are outlined. The penumbral branch P2 is divided into two parts. The region inside of the white box of the Ca II $H$ line-centre image is used to determine the mean Ca II $H$ brightness of the "quiet" Sun; the white box in the Ca II $H$ line-wing image marks the subregion shown in Fig. 12. Coordinates are given relative to the origin of Fig. 1.

consider the systematically decreasing image quality from the lock point of the adaptive optics system towards the borders of the frames, subfields with sizes of $128 \times 128$ pixels were reconstructed separately with different speckle transfer functions. The thus achieved cadence is $19 \mathrm{~s}$ between subsequent reconstructed images.

The slow scanning mechanism made the frame rate in the Ca II $H$ channel much smaller than in the other blue channels. To obtain statistical significance for applying speckle reconstruction, the corresponding data were bundled into packages of 36 frames. I.e., data from nine consecutive scans were merged. By applying this procedure, a cadence of $57 \mathrm{~s}$ between subsequent reconstructed images for each line position was achieved. In addition, a higher cadence (19s) was obtained during the flare eruption by speckle-reconstructing packages of only 12 frames (three consecutive scans). The results of this latter procedure are only marginally worse than those using the threefold number of frames.

Although, in principle, 125 images per polarisation state and line position should have been stored in the red beam, only a much lower number of images were fully free of interference, therefore, packages of 50 frames were bundled in the red channels. For the camera mounted behind the SOUP instrument, this corresponds to the 50 frames with the highest RMS-contrast at 


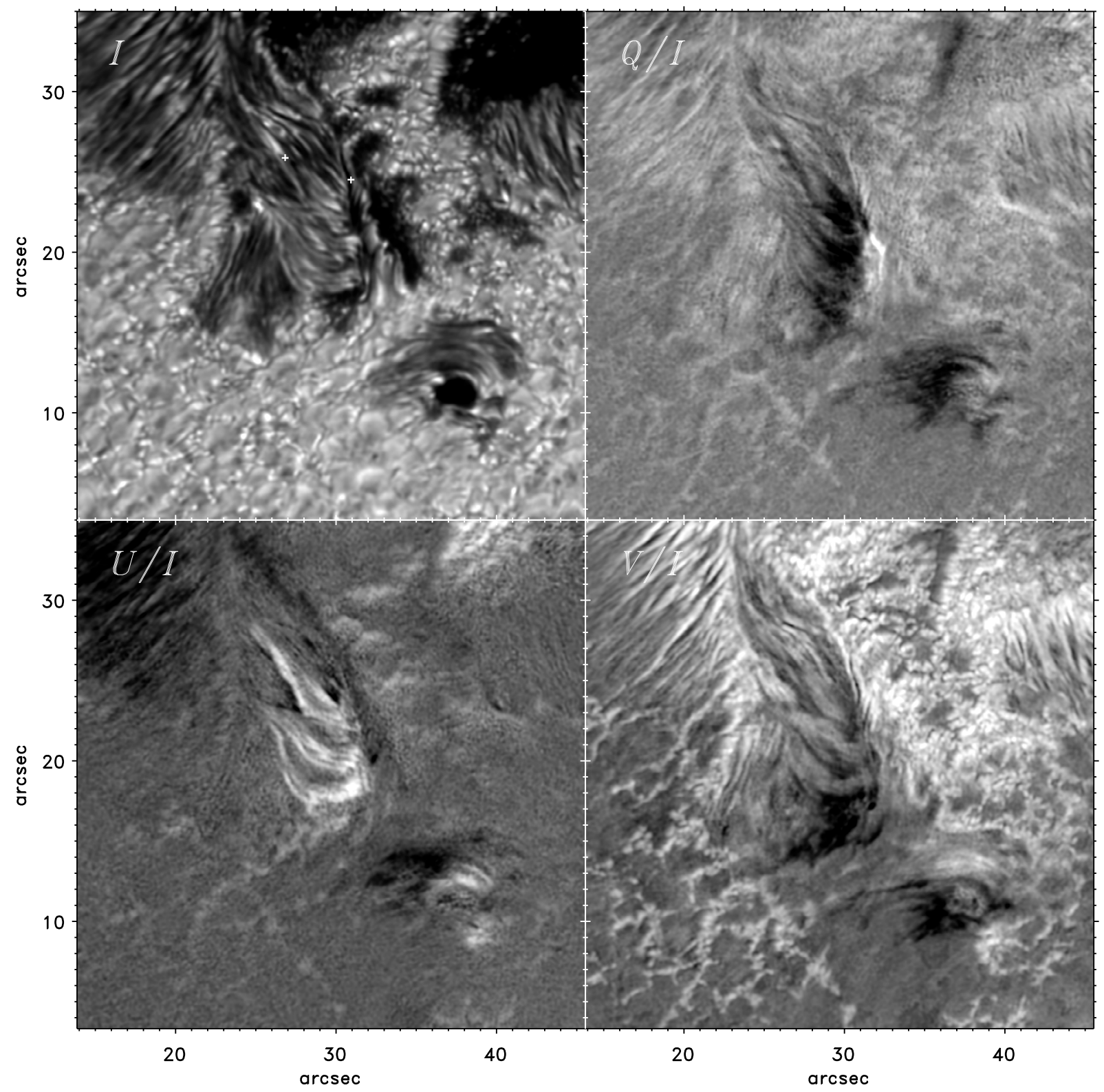

Fig. 3. Maps of the Stokes vector of the region of interest at $\lambda=\lambda_{0}-75 \mathrm{~m} \AA$. Grey levels have been scaled to the minimum and maximum values of each component. The "+" signs in the Stokes I image mark the positions of the Stokes profiles displayed in Fig. 4.

each line position and polarisation state. From the broad band channel, 50 frames obtained exactly simultaneously with the narrow band images were selected, and speckle reconstruction was applied. Figure 2 shows examples of reconstructed broad band images (red continuum and G-continuum), as well as $\mathrm{Ca}$ II $H$ line-core and line-wing images of the region harbouring the flare.

Reconstruction of narrow band data (SOUP channel) was carried out by implicitly calculating the optical transfer functions from the broad band data (see Krieg et al. 1999). This procedure yields a 4-component polarised light vector, $\boldsymbol{I}(x, y, t, \lambda)$, depending on the spectral position, $\lambda$, time, $t$, and image coordinates, $x$ and $y$.

\subsection{Polarimetric demodulation}

Instrumental polarisation effects of the laboratory setup were measured by inserting calibration optics into the beam and gradually rotating a linear polariser. From the thus obtained data the demodulation matrix, $\boldsymbol{M}_{\mathrm{lab}}$, was determined by using a code developed by Selbing (2005). The instrumental polarisation of the SST varies with the rotating field of view of the turret system. The corresponding demodulation matrices, $\boldsymbol{M}_{\mathrm{SST}}(t)$, were obtained by using a telescope model also provided by Selbing (2005). Subsequently, the Stokes vectors, $S^{\mathrm{T}}=(I, Q, U, V)$, were computed with

$\boldsymbol{S}(x, y, t, \lambda)=\boldsymbol{M}_{\mathrm{SST}}(t) \cdot \boldsymbol{M}_{\mathrm{lab}} \cdot \boldsymbol{I}(x, y, t, \lambda)$.

Examples of the resulting Stokes vectors are shown in Figs. 3 and 4 . The estimated $R M S$-polarimetric noise level, derived from a comaprison of the noise of Stokes $Q$ and $U$ with Stokes $I$ in the quiet continuum, is of the order of $1 \%$. 

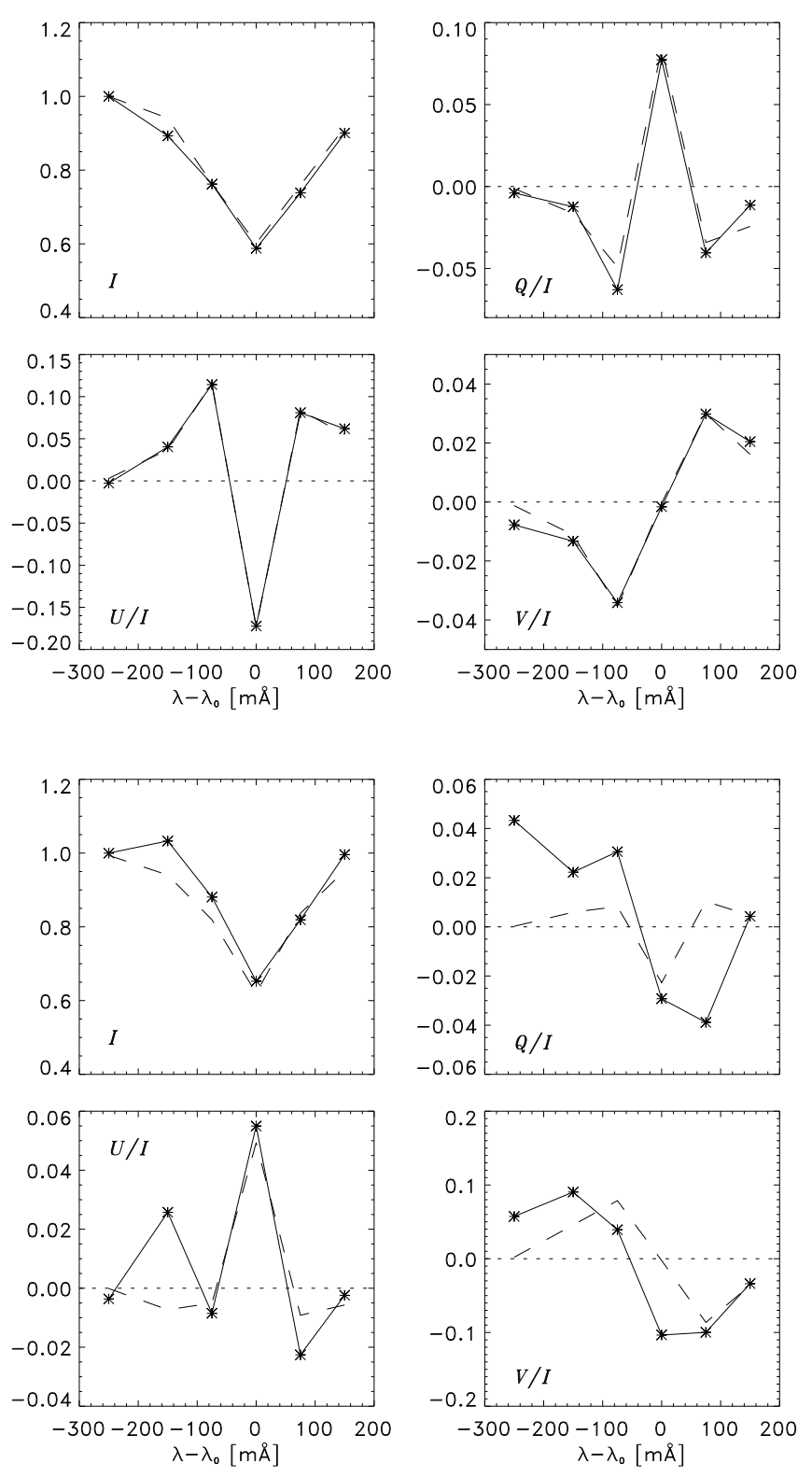

Fig. 4. Examples of regular and abnormal Stokes profiles after image reconstruction and demodulation as measured with the employed instrumental setup (solid) overplotted by the result of the Milne-Eddington inversion (dashed). The examples stem from a penumbral filament (upper four panels) and from a dark channel crossing the disturbed penumbra region (lower four panels; see "+" signs in Fig. 3). Remarkable is the strong Stokes $V$ signal at $\lambda=\lambda_{0}-250 \mathrm{~m} \AA$ in the lower example. This indicates an atmospheric component of strong upward mass flow. The one-component Milne-Eddington inversion yields a good approximation to the regular Stokes profiles in the upper example but fails to reproduce the abnormal profiles of the lower example. The impression of asymmetric Milne-Eddington profiles is caused only by the asymmetric sampling of the spectral line.

\section{Inversions of Stokes profiles}

To determine the magnetic field vector reliably, we inverted the radiative transfer equation (RTE) for polarised radiation in the Zeeman-split Fe I 6302.5 ̊ line. The small number of only six wavelength points in general give us 21 significant data points $\left(Q, U\right.$, and $V$ are usually close to zero at $\lambda_{0}-\lambda=-250 \AA$, see however Fig. 4) per spatial pixel, whereby in the penumbra all four Stokes parameters are well above the noise. So few observables can easily lead to unrealistic inversion results if the number of free parameters is not kept low.

We therefore inverted the data assuming a simple onecomponent-plus-straylight Milne-Eddington atmosphere using the "HeLIx" inversion procedure (Lagg et al. 2004). The model atmosphere has eight free parameters. These are the three components of the magnetic field vector $\boldsymbol{B}=(|\boldsymbol{B}|, \gamma, \chi)$ - where $\gamma$ denotes the field inclination to the line-of-sight (LOS) and $\chi$ is the field azimuth angle - the LOS flow velocity, $v_{\text {LOS }}$, the Doppler broadening, the amplitudes of the components of the propagation matrix and, finally, the geometric filling factor, $\alpha$. In addition, a straylight atmospheric component with a filling factor $(1-\alpha)$ was included into the model atmosphere. In this component the magnetic field vector is assumed to be zero, and all other parameters are coupled to the values of the magnetic component. To keep the number of free parameters small in the inversions, we abandoned fitting the constant term of the source function of the Milne-Eddingtton atmospheric model. It has been shown by Orozco Suarez et al. (2007) that this cobstraint introduces an ambiguity between the field strengths, $|\boldsymbol{B}|$, and the filling factor, $\alpha$, i.e. profiles produced from regions with high field strength and low filling factor often cannot be distinguished from those of low field strength and high filling factor. Therefore, we henceforth use the term "field strength" synonymously with the flux density, $|\boldsymbol{B}| \cdot \alpha$.

Selected model parameters obtained for the region of interest are shown in Fig. 5. Direct interpretation of these parameter maps is hindered by the inevitable $180^{\circ}$-ambiguity of the magnetic field azimuth and by the magnetic field vectors being given in a coordinate system that is inclined to the local coordinate system by the heliocentric angle. However, to reliably transform the field vectors into a local coordinate system the azimuth ambiguity has to be resolved. As can be seen in Fig. 5, two large regions are obviously afflicted with an erroneous azimuth direction. In some other areas of the region of interest, the "true" azimuth direction is less evident. Common procedures to resolve this problem (see e.g. Metcalf et al. 2006) are often based on additional assumptions, e.g. potentiality or minimum energy configurations, on the magnetic field structure, which are not fulfilled in a flaring region. We, therefore, tried to resolve the azimuth ambiguity by inverting the azimuthal direction in those regions where it is obviously wrong and then iteratively minimising the gradients of the field azimuth in the region of interest (see also Zakharov et al. 2008). This procedure works in a similar fashion to the AZAM code described by Lites et al. (1995). In Fig. 6 we display the magnetic field vector after resolution of the azimuth ambiguity and after transformation into a local coordinate system where the $z$-direction is perpendicular to the solar surface.

\section{Results}

Solar active region NOAA 10904 is a bipolar sunspot group comprising two mature spots, one at each magnetic polarity. The observed trailing spot shows mainly positive polarity (see Fig. 23) and contains tiny regions of inverse (negative) polarity in its outer parts. Although this spot includes both polarities, it might be classified as a part of a $\beta$-group (Potsdam classification, cf. Künzel 1960) rather than as a $\delta$-spot, but see, e.g., Lites et al. (1995) and references therein for a discussion about the formation of $\delta$-spots. While observing the sunspot, a flare erupted in the disturbed penumbral region at its disc-centre side. The flare started at about 8:47 UT and lasted only about ten minutes. The flare was also registered by the Solar X-ray Imager 


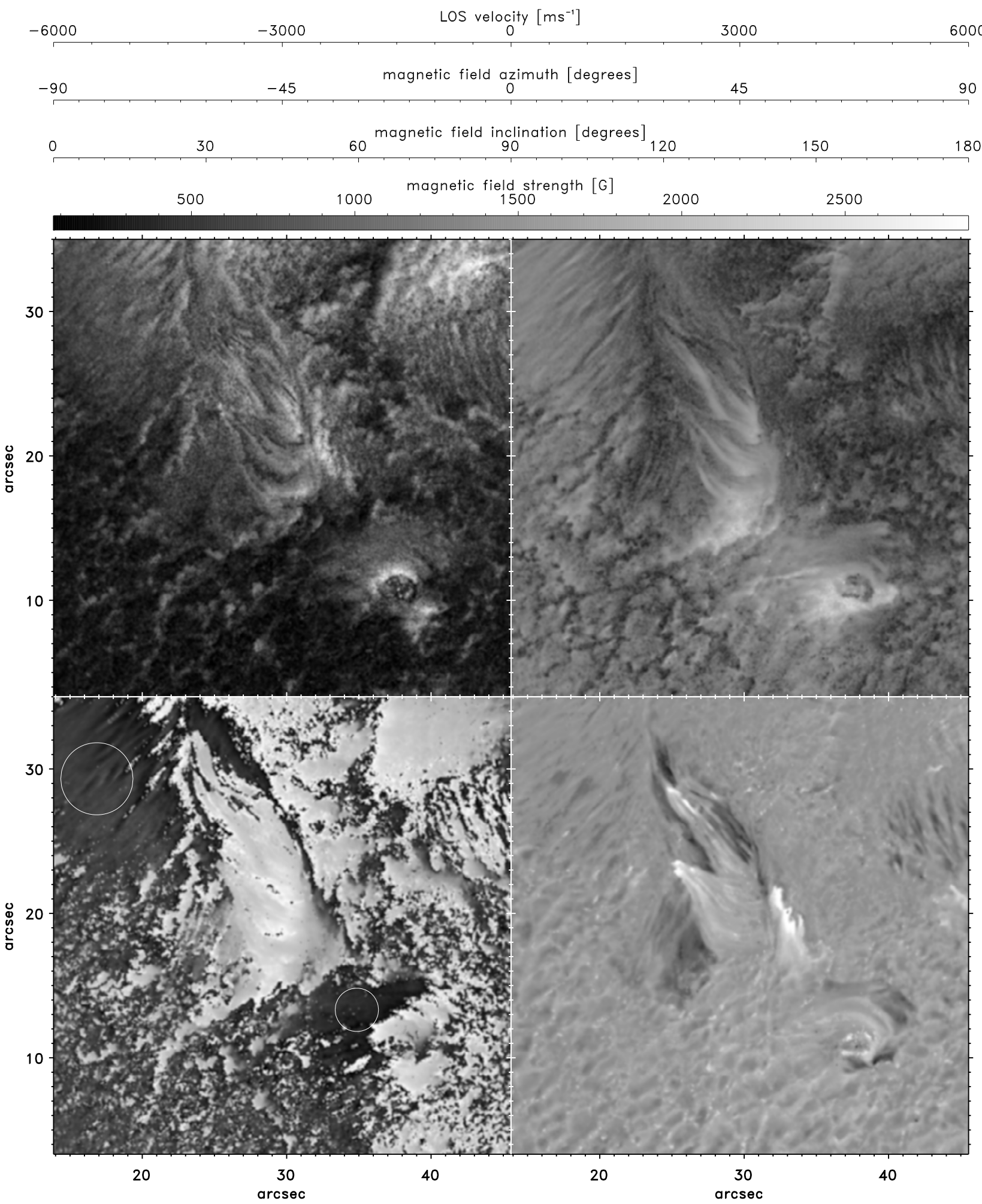

Fig. 5. Results of a one-component Milne-Eddington inversion of the Stokes profiles measured between 8:41:43 UT and 8:43:24 UT. Upper left: magnetic field strength; upper right: field inclination relative to the LOS; lower left: field azimuth (perpendicular to the LOS); lower right: LOSvelocity. The regions marked with circles are in all likelihood suffering from an $180^{\circ}$ error of the field azimuth.

(SXI) onboard NASA's GOES-12 satellite. The SXI data show an emission peak at 8:53:00 UT reaching a magnitude of B7.8. In Fig. 2 the disturbed penumbral region is displayed just before the start of the flare. In the red continuum image, several penumbral branches and small umbrae are highlighted. These features are, henceforth, called "P1" to "P4" and "U1" to "U4", respectively.

\subsection{Photospheric flow morphology}

Determination of the photospheric flow structure is limited by the ability of only the LOS component being derived directly from the Doppler shifts of spectral line profiles. The transversal flow components can only be estimated by tracking the motions 


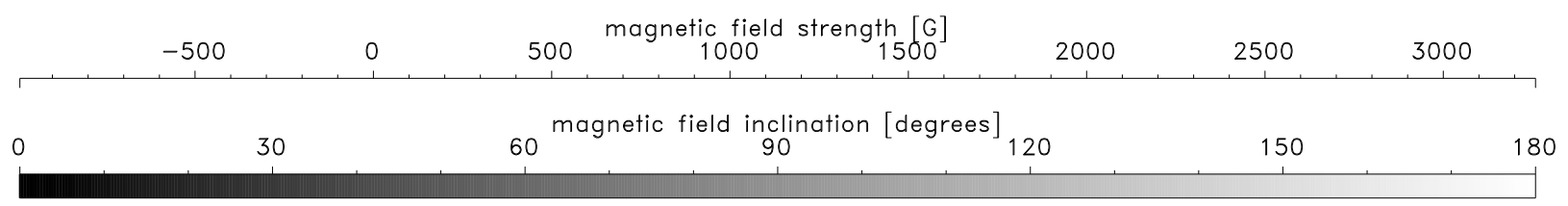

$-180$ $-90$ magnetic field azimuth [degrees]

90 180

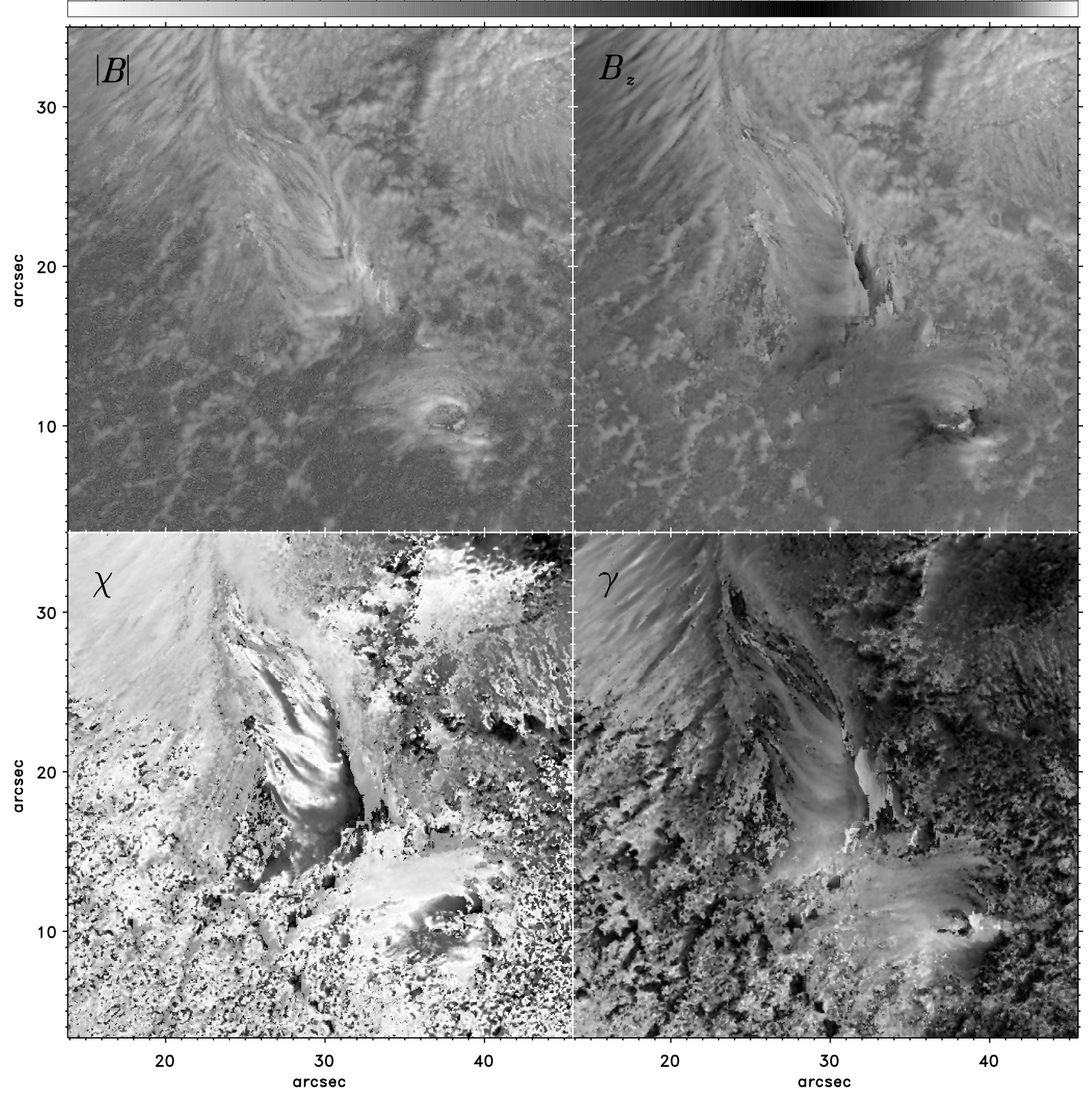

Fig. 6. Results of a two-component Milne-Eddington inversion of the Stokes profiles measured between 8:41:43 UT and 8:43:24 UT after transformation into a local coordinate system where $z$ is perpendicular to the solar surface and $x$, i.e. $\chi=0$, is directed towards the right. Upper left: $|\boldsymbol{B}| \alpha$, upper right: $B_{z}$, lower left: $\chi$, lower right: $\gamma$.

of intensity structures, which might be misled by moving intensity patterns, such as waves, which are not real mass flows. In the left panel of Fig. 7, the mean horizontal flow velocities in the flare region is displayed, estimated from a $33 \mathrm{~min}$ time series of G-continuum images. The flows have been calculated using the local correlation tracking technique (LCT, cf.
November \& Simon 1988) with a Gaussian window function of one arcsecond width.

Close to the main umbra of the sunspot the flow field shows the well-known penetration of penumbral grains into the umbra (see e.g. Sobotka \& Sütterlin 2001; Bovelet \& Wiehr 2003). A conspicuous feature in the disturbed penumbra is a fast 

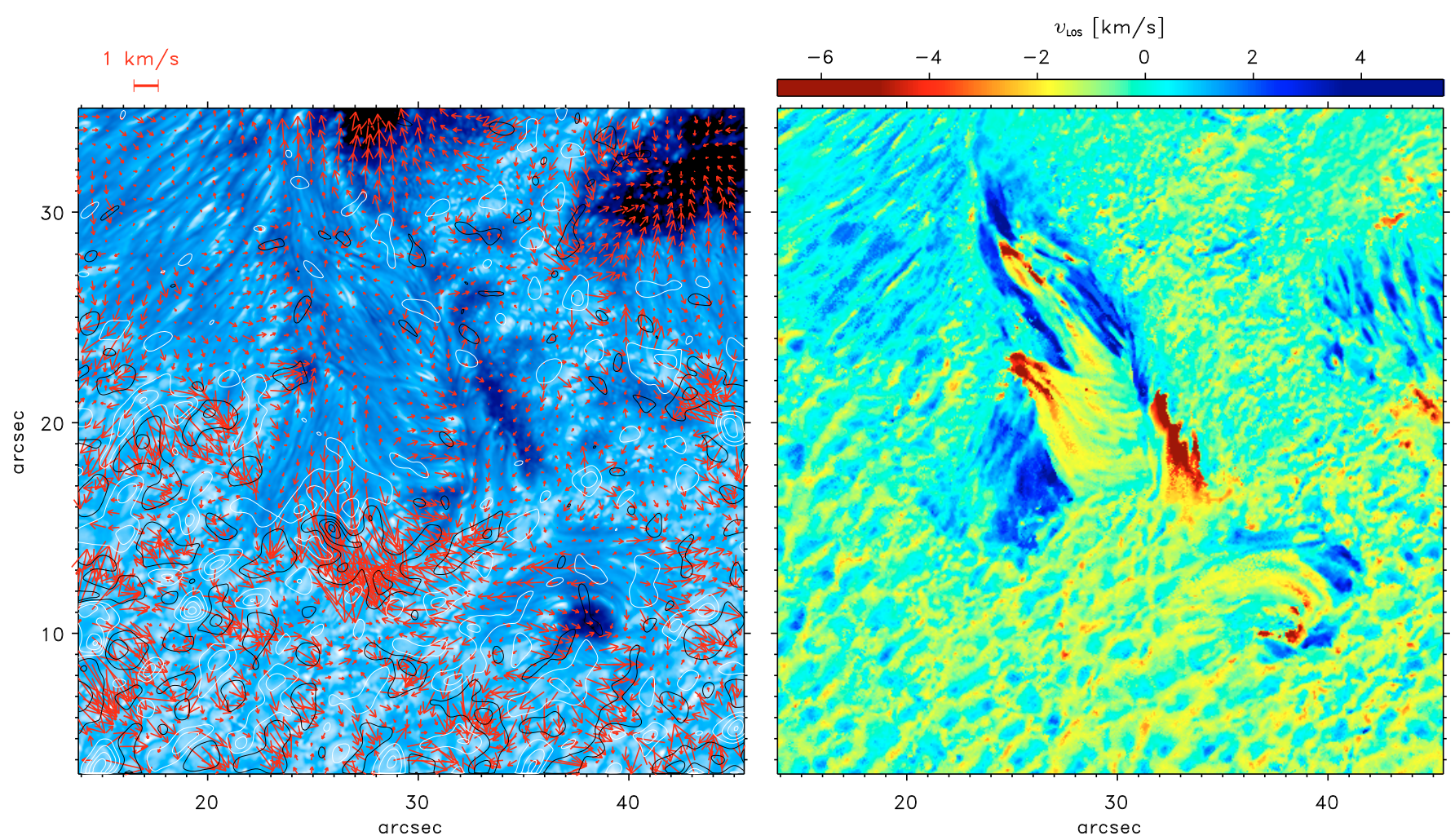

Fig. 7. Photospheric flows in the flaring region. Left panel: time-averaged horizontal flows from a series of 106 G-continuum images obtained between 8:28:37 UT and 9:01:44 UT; contour lines represent the divergence of the horizontal velocities (white contours encompass regions of positive divergence, negative divergence regions are located within black contours) ${ }^{1}$. Right panel: line-of-sight flow velocities obtained from a Milne-Eddington inversion of a line scan lasting from 8:43:47 UT to 8:45:31 UT.

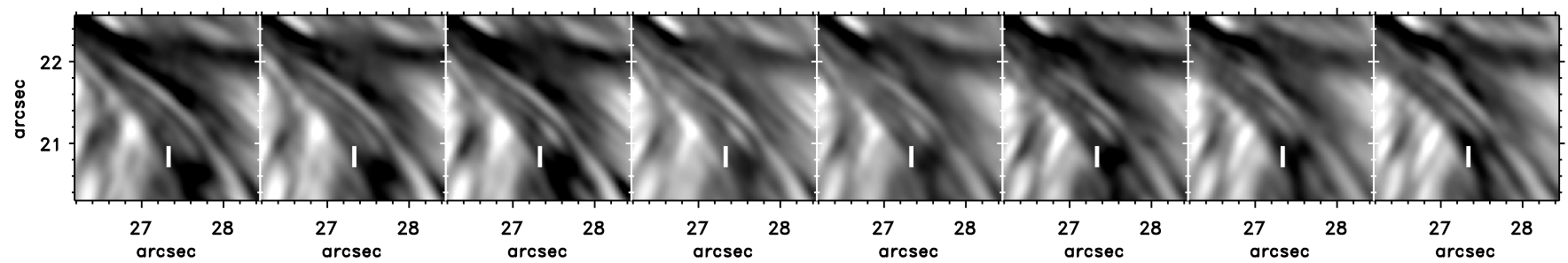

Fig. 8. Fast motion of a fragment of a penumbral fibril located above the white reference marks: The figure represents a time series of G-continuum images between 8:38:03 UT and 8:40:15 UT.

penetration into the adjacent quiet region at $\left[26^{\prime \prime}, 16^{\prime \prime}\right]$. There the derived horizontal flow speeds achieve values up to $2.1 \mathrm{~km} \mathrm{~s}^{-1}$. The nearby quiet region [26", $\left.15^{\prime \prime}\right]$ marks a position of strong flow convergence. In the penetrating penumbral branch (henceforth called P2, cf. Fig. 2) itself (at [27"'5, 17"]), a divergence centre can be detected. The fast horizontal velocities are not artifacts produced by the LCT procedure since the penumbral outflow can be easily visualised by an animation of the time series of G-continuum images, which is available as an accompanying mpeg-movie. The concomitance of the outward flow with continuous positive Doppler shifts (see right panel of Fig. 7) of up to $3 \mathrm{~km} \mathrm{~s}^{-1}$ suggests fast-moving Doppler clouds that are clearly visible in a though discontinuous animation of the available Dopplergrams. The derived velocities of up to $2.1 \mathrm{~km} \mathrm{~s}^{-1}$ are well within the range of the proper motion speeds of Evershed clouds reported in Cabrera Solana et al. (2007).

In addition to the convergence centre at $\left[26^{\prime \prime}, 15^{\prime \prime}\right]$, other centres of horizontal flow convergence and divergence, which can be associated with outward and inward motions, can be found all around the disturbed penumbra. The derived flow velocities are mainly low within the disturbed penumbra. One exception is the region around [31."5, 19"] where several bright heads of penumbral fibrils (outlined as P1 in Fig. 2) move with flow velocity of up to $375 \mathrm{~m} \mathrm{~s}^{-1}$ towards the adjacent penumbral branch (P4), marking another centre of flow convergence. Other exceptions are the convergence centres at [26."5, 28."5] (close to the upper end of branch P3) with maximum inflow speeds of about $250 \mathrm{~m} \mathrm{~s}^{-1}$, and the region left to the small umbra U3 $\left[23 . " 5,22^{\prime \prime}\right]$ with maximum flow speeds of $480 \mathrm{~m} \mathrm{~s}^{-1}$.

LCT can provide only temporally averaged and smoothed (by the used window function) flows. However, inspection of a fast animated movie of the time series reveals a wide variety of dynamic features that are not visible in the LCT flow map; e.g., at the crossing region of penumbral branches P1 and P2 (at $\left[27 . " 5,21^{\prime \prime}\right]$ ), the LCT map shows a weak flow in $y$-direction.

\footnotetext{
1 An accompanying animation of the G-continuum images is available online at http://www . aanda. org
} 

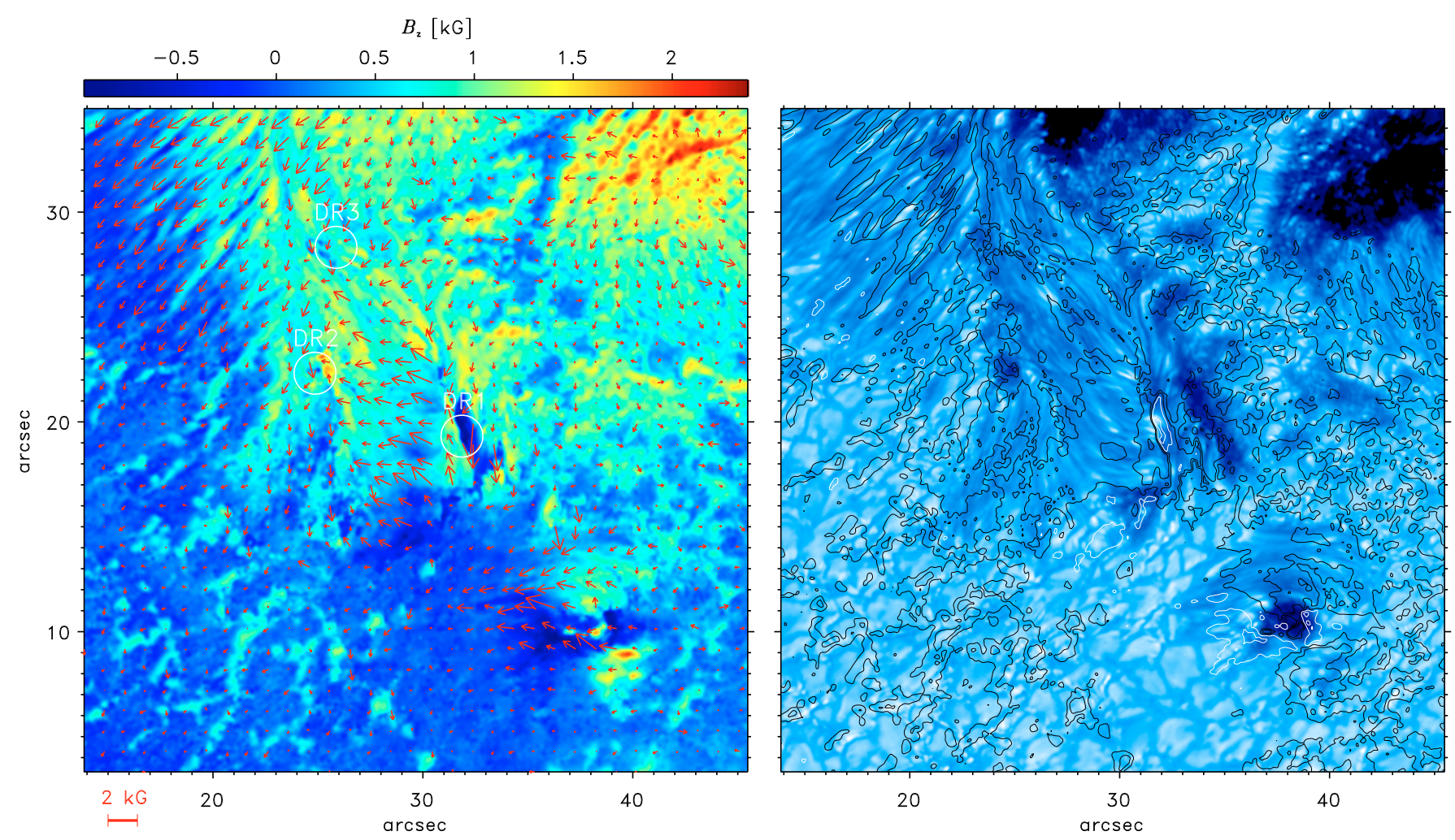

Fig. 9. Magnetic field configuration in the flare region. Left panel: vertical magnetic field map $\left(B_{z}\right)$ overplotted by vectors showing the horizontal field structure $\left(B_{x}, B_{y}\right)$ obtained from an inversion of the line scan between 8:43:47 UT and 8:45:31 UT. Right panel: G-continuum image from 8:44:40 UT overplotted with $B_{z}$-contour lines at $-750,-250,250$, and $750 \mathrm{G}$.

However, the corresponding G-continuum movie shows a fast motion of the lowermost fibrils of branch P1 towards the dark umbra U3 located at [25", $\left.22^{\prime \prime}\right]$; i.e., they move at an angle of more than $45^{\circ}$ to the mean flow in that region. This motion is illustrated in Fig. 8, which shows a time series of 2". $25 \times 2$ ". 25 excerpts of eight consecutive G-continuum images. Therein the motion of a fragment of a penumbral fibril can be followed. Within the first 6 images, the structure moves about 0 ".25, which corresponds to a velocity of $1.9 \mathrm{~km} \mathrm{~s}^{-1}$. Later on, the identification of the structure becomes difficult. For comparison, penumbral grains move with mean speeds between $0.5 \mathrm{~km} \mathrm{~s}^{-1}$ and $0.8 \mathrm{~km} \mathrm{~s}^{-1}$ (Sobotka \& Sütterlin 2001).

As obtained from a one-component Milne-Eddington inversion of a line scan just before the impulsive phase of the flare, the LOS velocities, $v_{\text {LOS }}$, are shown in the right panel of Fig. 7. Flows towards the observer are assigned to positive velocity values. The entire disturbed penumbra is interspersed with exceptionally high velocities both towards (upflows) and away from the observer (downflows). In several regions, the downflows reach almost $v_{\mathrm{LOS}}=-7 \mathrm{~km} \mathrm{~s}^{-1}$, which might be higher than the local sound speed in these regions. The detected motions in the disturbed penumbra might be attributed to Evershed flows. Thus, the different branches of the disturbed penumbra are characterised by Evershed motions that point in a direction specific to each branch. Particularly interesting is the branch P1 (located around $\left.\left[27^{\prime \prime}, 22^{\prime \prime}\right]\right)$ that is associated with strong redshifts. It intersects another branch (P2) ranging from [24", 30"] down to $\left[25^{\prime \prime}, 13^{\prime \prime}\right]$. Therfore, $\mathrm{P} 2$, which is associated with strong blueshifts, is divided into two parts (see Fig. 2). Another interesting feature is the redshifted branch P3 that intersects a large blueshifted area around [25",27"]. Finally, one more region associated with possibly supersonic downflows is branch P4, located around [33", 19"]. This branch corresponds to a penumbral region consisting of short diffuse fibrils located between an elongated umbral structure (U4) and a dark lane that divides P4 from P1 and P3. Surprisingly, only the lower part of P4 is redshifted, and its upper part shows blueshifts of several kilometres per second. A strikingly twisting penumbral filament (see Ichimoto et al. 2007; Zakharov et al. 2008) is located at the transition from red- to blueshifts .

Supersonic flow motions in disturbed penumbral regions or in the normal penumbra are well-documented (see Martínez Pillet et al. 1994, and references therein). These authors report photospheric flow velocities (downflows) of up to more than $14 \mathrm{~km} \mathrm{~s}^{-1}$, close to the magnetic inversion line of a $\delta$-spot. Lites et al. (2002) found velocities of about 6 to $8 \mathrm{~km} \mathrm{~s}^{-1}$ in a broad region between the magnetic inversion line of a $\delta$-spot and the nearby border where upflows are separated from downflows. They hypothesised an explanation for this result by assuming a region of interlaced penumbral fibrils of different penumbral branches. Bellot Rubio et al. (2007) have found evidence that the Evershed flow is supersonic at many locations in the penumbra, confirming earlier hints (e.g. Borrero et al. 2005).

Summarising the discussion of the features visible in the flow maps, it might be stated that both horizontal and LOS velocities show strong fluctuations within short length scales. This means that the flows in the entire disturbed penumbra seem to be strongly sheared.

\subsection{Magnetic field structure}

The magnetic field structure of the flare region is displayed in Fig. 9. The Cartesian components of the magnetic field 

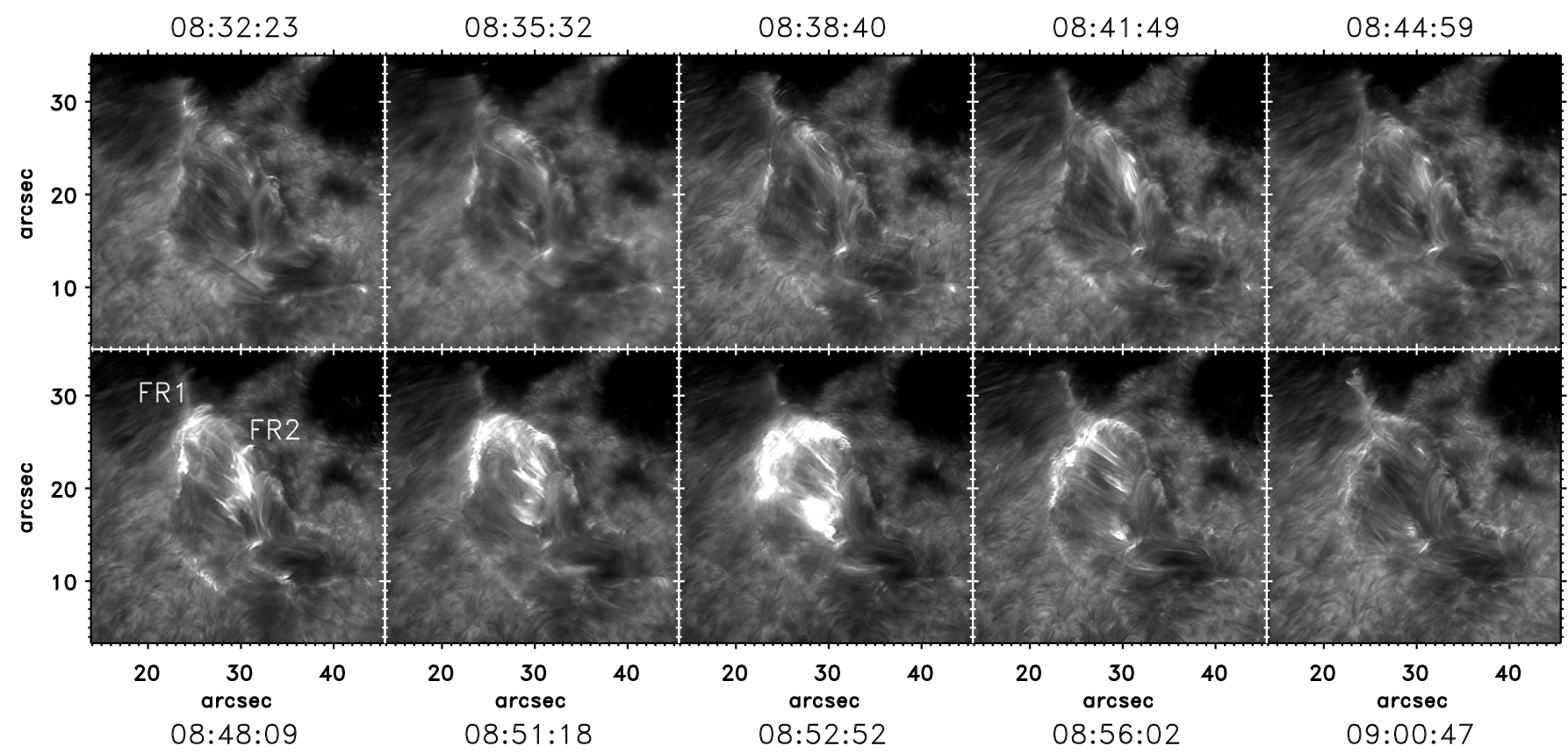

Fig. 10. Evolution of the CaII $H$ line core intensity during the flare eruption above the disturbed penumbra region. Clock time labels are given in UT. The grey code is the same for all images ${ }^{2}$.

vector $\left(B_{x}, B_{y}, B_{z}\right)$ are shown after resolving the azimuth ambiguity and transformation into a local horizontal coordinate system. We stress that a resolution of the azimuth ambiguity was not possible without leaving discontinuities of the field azimuth. The applied procedure only provides a best guess of the horizontal field direction obtained by keeping the discontinuity regions as small as possible. Figure 9 shows discontinuities at [32", 19".5] (DR1) and (less pronounced) at [25", 22".5] (DR2). In the right panel of Fig. 9 it can be seen that DR1 coincides with a neutral line of $B_{z}$, separating a small region of negative (downward) vertical field from the surrounding regions of positive (upward) $B_{z}$. The second discontinuity region (DR2) is located at the right border of the small umbra U3.

Penumbral branch P1 (see Fig. 2) connects these two magnetic field discontinuities and carries fast negative Evershed motion from DR1 to DR2 (see Fig. 7). The magnetic field in P1 is mainly horizontal and directed towards DR2. This is almost perpendicular to the radial direction of the sunspot. A close inspection of the Doppler map and the G-continuum images (see Fig. 7) indicates that this branch crosses penumbral branch P2 at the position of DR2. The underlying branch P2 carries a fast positive Evershed flow that is directed more or less radially outwards. Also the field vectors point nearly radially outwards. Thus, this branch might be considered as the "normal" spot penumbra that is intersected by $\mathrm{P} 1$, and $\mathrm{P} 3$ is another branch of penumbral filaments carrying negative Evershed flows (located between $\left[26^{\prime \prime}, 28^{\prime \prime}\right]$ and the flow convergence region at $\left.\left[31^{\prime \prime}, 19^{\prime \prime}\right]\right)$. This might be a sub-branch of P1 that also intersects the global structure of the sunspot penumbra. Again the magnetic fields in this branch are in opposite directions to the global fields of the sunspot. A convergence centre of the horizontal flows is located at the upper end of this branch (i.e. at $\left.\left[26^{\prime \prime}, 28^{\prime \prime}\right]\right)$. Also the horizontal magnetic field is somewhat noisy (therefore, we call this region DR3, henceforth), although a clear discontinuity cannot be detected. This noisy behaviour come from the fact that a Milne-Eddington inversion assumes a height-independent magnetic field, which is most likely not valid in a region of crossing penumbral branches.

The description of the global magnetic field structure in the disturbed penumbra region can be summarised as the fields being largely co-aligned with the Evershed flows. This leads to discontinuities in the horizontal fields that are co-spatial with the convergence points of the Evershed flows and also of convergence centres of the horizontal flows (proper motions of penumbral structures). In addition, several islands of oppositely (downward) directed vertical fields are present within and around the disturbed penumbral region. This configuration is largely comparable to the magnetic field configuration of the flare model developed in Falconer et al. (2000).

\subsection{Ca II H observations}

The evolution of the flare with time as seen in the Ca II $H$ line centre images obtained with the SST is shown in Fig. 10. An animation is available as accompanying mpeg-movie. The preand post-flaring region above the disturbed penumbra is easily discernible (see also Fig. 2). It is darker than the adjacent rather quiet region, visible in the lower left corner of the images displayed in Fig. 10 and also darker than the disturbed granular region, which appears between the disturbed penumbra and the two umbrae of the spot. The border between the pre-flaring region and its surroundings shows enhanced brightness in the line core of $\mathrm{Ca}$ II $H$. This bright border might be interpreted as the intersection of a separatrix dividing the magnetic fields inside the disturbed penumbra from its surroundings with the chromospheric level where the calcium line is formed (see Falconer et al. 2000 and below).

Inside the region enclosed by the separatrix the $\mathrm{Ca}$ II $H$ line core images show a jumble of long fibrils that are mostly connected to different parts of the bright border or to small-scale

\footnotetext{
2 An accompanying animation of the Ca II $H$ line core images is available online at http://www . aanda.org
} 
bright structures inside this region. These structures might be closely related to the "straws" described by Rutten (2007) and the Ca II $K$ fibrils recently discovered by Pietarila et al. (2009). This appearance is significantly different from the normal penumbra at the limb-side of the sunspot. There the fibrils are radially oriented, i.e. mainly parallel to the photospheric penumbral fibrils. The normal Ca II $H$ line core penumbra is also rougher-textured and - on average - darker than the disturbed flare region (see Fig. 24 for a Ca II $H$ line core image of the entire field of view of the observations.).

As in a mature two-ribbon flare, the eruption starts simultaneously at two stripes, which are separated by approximately 7 to 8 arcsec (see lower left panel of Fig. 10). One of the two ribbons (FR1) appears first just at the separatrix and extends later on to an opposite part of the separatrix, marking the inner penumbral boundary (sometimes called peripatopause) between the disturbed penumbra and the larger sunspot umbra. Two branches of fibrils originally cross in the region of the second flare ribbon (FR2). One of these branches flashes up and expands until it merges with FR1 (see panel from 8:48:09 to 8:52:52 UT). During the rapid expansion of the upper part of FR2 (see panel from 8:51:18 UT), the flashing fibrils form an arcade of bright streaks at the upper part of the flaring region. This arcade is located between the upper ends of the two flare ribbons. Its upper footpoints are co-spatial with the separatrix region. Later on, the centre of the flare brightness moves toward the lower ends of the flare ribbons (see panel from 8:52:52 UT) and the maximum brightness seems to be emitted by long thin fibrils crossing and enclosing both flare ribbons, as well as from their footpoints at FR1. The width of these fibrils is only about 3 to 4 pixels, i.e. about 0 .' 15 although their length reaches values up to 10 arcsec. These values are in good agreement with the dimensions of recently discovered Ca II $K$ fibrils seen in plage regions (Pietarila et al. 2009). After 8:52 UT, the flare brightness slowly decreases until the region reaches a similar state as during its pre-flare phase at approximately 9:00 UT.

Unfortunately, the total intensity flux of the flare could not be measured since the detector was saturated during the impulsive phase of the flare. Instead of that, we estimated the evolution of the total flaring area (see Fig. 11). This area is defined as regions achieving intensities more than double that of the average quiet Sun Ca II $H$ line core intensity estimated from a region to the lower left of the flaring region (see Fig. 2). The flaring area reaches its maximum extent of $30.94 \mathrm{Mm}^{2}$ at 8:52:52 UT. Its rising phase lasts slightly longer than its decline and, as already mentioned, shows a few bumps. In general, the shape of this curve is similar to that of the GOES measurements although only one smooth peak is visible in the GOES-12 X-ray measurements. GOES-8 X-ray data show a bump in the declining phase of the flare.

The effects of the flare eruption on the blue wing of Ca II $H$ are shown in Fig. 12. An animation is available as an accompanying mpeg-movie. The first indication of the flare appears at 8:47:12 UT as an isolated bright filamentary structure (in Fig. 12 at position [22".5, 22."5]). At 8:48:09 UT another bright structure appears at $[26 . " 5,18 . " 5]$, which seems to be an extension of the one seen before. Finally, as the flare reaches its maximum brightness in the line centre, a definite loop structure becomes visible. Its footpoints are rooted in rather close proximity to each other. One of the footpoints is associated with a bright flash at 8:52:52 UT, whereas the other one shows no brightness enhancement until 8:53:49UT. Later on, another bright structure appears at $8: 54: 46$ UT right between the two footpoints at $\left[27.15,21^{\prime \prime}\right]$.

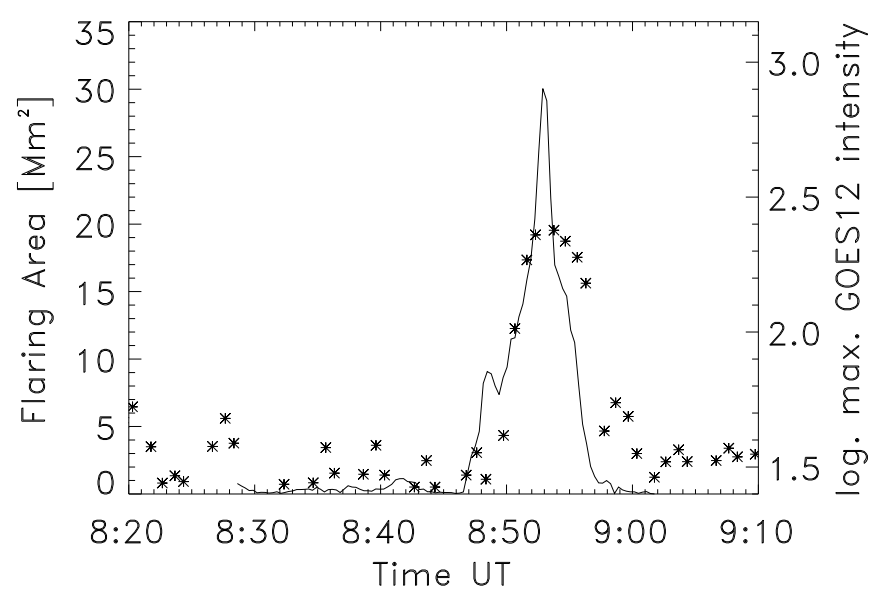

Fig. 11. Temporal variation of the flaring area, which is defined by those regions that are brighter than twice the mean quiet Sun $\mathrm{Ca}$ II $H$ line core brightness (solid). The asterisks show the logarithmic maximum SXI/GOES12 intensity of the flaring region (arbitrary units).

\subsection{Relation of magnetic fields and the flare ribbons}

To demonstrate that the determined magnetic field structure is realistic, the horizontal magnetic field is overplotted in Fig. 13 on a $\mathrm{Ca}$ II $H$ line core image at the onset of the flare eruption. The region of magnetic azimuth discontinuity DR 1 at $\left[32^{\prime \prime}, 19\right.$ ".5] is located to the right of the onset region of FR2. The onset of FR1 takes place above the small umbra U3, leftward of DR2. Figure 13 shows that region DR3 also participates in the flare since it is located slightly to the right of the upper end of FR1. The displacement between the discontinuity regions and the flare ribbons might be (partially) explained by a parallax caused by the inclined line of sight. The displacement of the discontinuities from the local flare intensity maxima is approximately one arcsecond in all three regions. This implies a (reasonable) difference of $1100 \mathrm{~km}$ between the height where the photospheric magnetic field information is obtained and the height of the brightest Ca II $H$ line core flashes.

\subsection{The onset of the flare}

The first appearance of the flare takes place in a region of two intersecting branches of Ca II $H$ line-core fibrils. In Fig. 14 the evolution of this region (FR2) as seen in the Ca II $H$ line core and in the blue continuum is displayed. After its first appearance, the fine structure of FR2, as displayed in Fig. 14, shows a series of bright loop-like structures (at 8:50:40 UT), which connect the position of their first appearance with a previously dark region. These loops expand rapidly and move towards the second flare ribbon. After that, the region of maximum Ca II $H$ brightness (cf. Fig. 10) spreads towards the lower left corner of the cutouts shown in Fig. 14.

The chromospheric structure in this region cannot only be characterised by two crossing branches of fibrils: In the blue wing of the Ca II $H$ line (see Fig. 12, panel for 8:52:52 UT) the onset region of the flare is connected with the lower end of the flare ribbon (i.e. with the small umbra (U2) in the lower left corner of the details shown in Fig. 14) via an extended loop. Since this loop is not clearly visible in the $\mathrm{Ca}$ II $H$ line-centre images, it must be either low-lying and/or become apparent due to strong Doppler shifts. Only moderate dynamics occur in the blue 


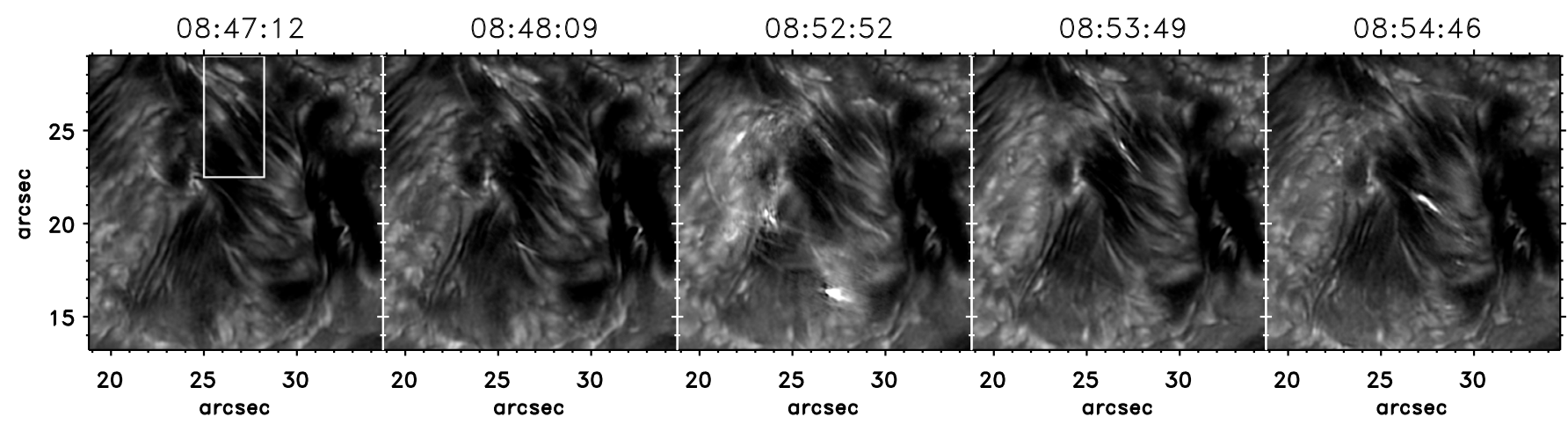

Fig. 12. Evolution of the Ca II $H$ wing intensity in a subregion (see Fig. 2). The white box marks the location of the detail shown in Fig. $20^{3}$.

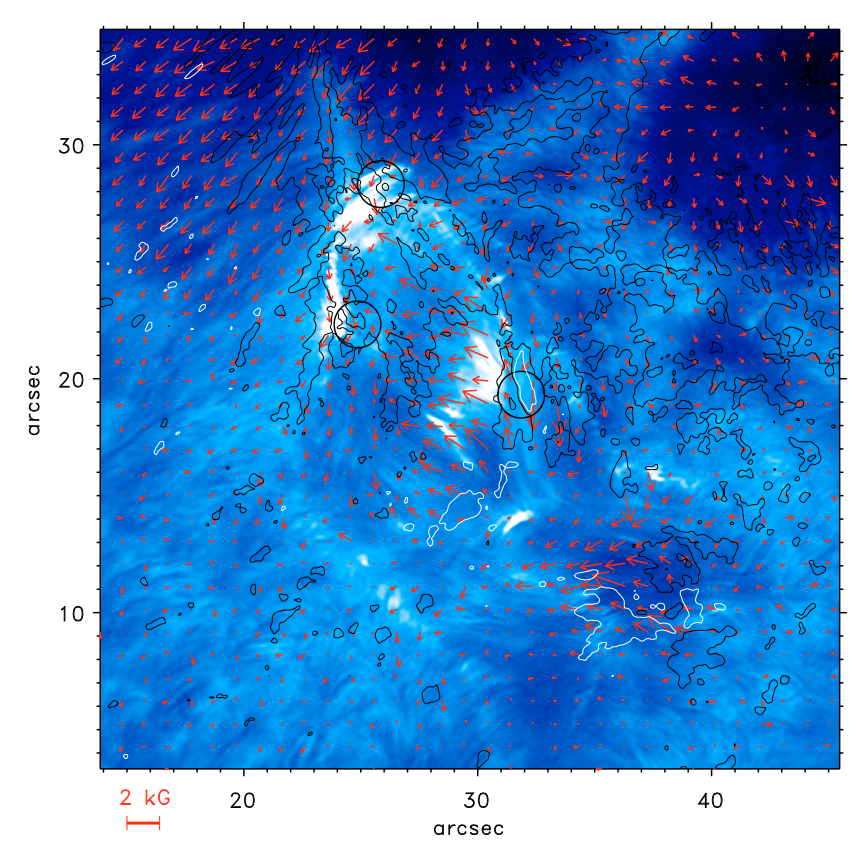

Fig. 13. Horizontal magnetic field (arrows) and vertical magnetic field (contours at -250 and $750 \mathrm{G}$ ) overplotted to the $\mathrm{Ca}$ II $H$ line-core intensity obtained at 8:47:31 UT at the onset flare eruption. Black circles mark regions of magnetic azimuth discontinuity (cf. Fig. 9).

continuum. The most conspicuous phenomena are two fast moving and evolving penumbral filaments (Fig. 14).

The corresponding magnetic field configuration is displayed in Figs. 15 and 16. Because of technical problems with the SOUP, magnetic field maps are available only shortly before (at 8:44:40 UT) and shortly after (at 8:53:50 UT) the flare onset. The most conspicuous feature in this region is a sharp reversal of the sign of $B_{z}$ in the centre of the cutouts shown in Fig. 15. This situation is not resolved by the flare eruption. Surprisingly, the island of negative $B_{z}$ grows during the flare eruption. This growth takes place mainly in the lower part of the displayed details where a penumbral filament (see lower white arrow in Fig. 14) moves into the small umbra U2. Throughout the entire observed period the magnetic neutral line separates penumbral branch P1, which carries fast Evershed motion from DR1 to DR2, from the island of negative $B_{z}$ which appears as a diffuse penumbral region (P4) in the blue continuum images. According to the moving flux tube model (cf. Schlichenmaier et al. 1998, but see, however,

\footnotetext{
${ }^{3}$ An accompanying animation of the Ca II $H$ line wing images is available online at http://www. aanda. org
}

Rempel et al. 2009) for penumbral filaments, the bright heads of the fibrils, which are located just leftward of the neutral line, appear where vertical flux tubes bend over and become horizontal. Thus, the field lines are mainly vertical in this region and at least some become more inclined, i.e. $B_{z}$ decreases and the horizontal field component, $B_{\mathrm{h}}=\left(B_{x}^{2}+B_{y}^{2}\right)^{1 / 2}$, increases, in about one arcsecond distance from the neutral line. Also the Doppler maps (see Fig. 7) show blueshifts in the region close to the neutral line, although the biggest fraction of this penumbral branch is characterised by strong redshifts.

A similar situation is present just rightward of the neutral line. In the magnetic island the $z$-component is strongest in close proximity to the neutral line and $B_{\mathrm{h}}$ achieves values up to $2.5 \mathrm{kG}$ at a distance of 0.5 to 1 arcsec from the neutral line. In the dark umbral region (U4) rightward of the magnetic island, $B_{z}$ is again strong (however positive) and $B_{\mathrm{h}}$ drops to values around $500 \mathrm{G}$. Therefore, it might be concluded that the diffuse penumbral region (P4) contains loop-like magnetic fields which emerge in the dark elongated umbra (U4) that is located in the upper right part of the cutouts shown in Figs. 14-16 and submerge in the island of negative $B_{z}$ close to the bright heads of the penumbral filaments of P1. Thus, the magnetic neutral line represents a region of strong vertical (and also horizontal) magnetic shear. We stress that the entire region of FR2, including the island of negative $B_{z}$, is located inside the separatrix appearing in the $\mathrm{Ca}$ II $H$ images. In fact, the separatrix is co-spatial with U4.

The onset of the other flare ribbon (FR1) is displayed in Fig. 17. Figures 18 and 19 show the corresponding magnetic field configuration. The flare onset takes place above the small roundish umbra U3 (visible in the middle of lower row frames in Fig. 17) and spreads out in both directions along the ribbon. The Ca II $H$ line-core images show some loop-like fibrils which connect U3 with the region around DR3. Again the photospheric structure shows only moderate dynamics. The magnetic field structure at the time of the flare onset (8:44:40UT) also does not show many conspicuous features. Worth mentioning are only two tiny regions of negative $B_{z}$ at the right border of the small umbra and close to DR3 (at [25".5, 28"]). The right panels of Figs. 18 and 19 show the field configuration approximately $40 \mathrm{~min}$ after the flare eruption. It can be clearly seen that the field structure has changed dramatically. Mainly the two regions of negative $B_{z}$ have become much larger and also the penumbral branch below the small umbra (lower part of P2) mainly comprises negative $B_{z}$. Simultaneously with the growth of the regions of negative $B_{z}$, in the same regions the horizontal field strength strongly increases up to values of more than $2 \mathrm{kG}$.

The scenario developed from analysing the Doppler maps and the general field topology in the region of interest can be substantiated and extended now. In this region several penumbral 


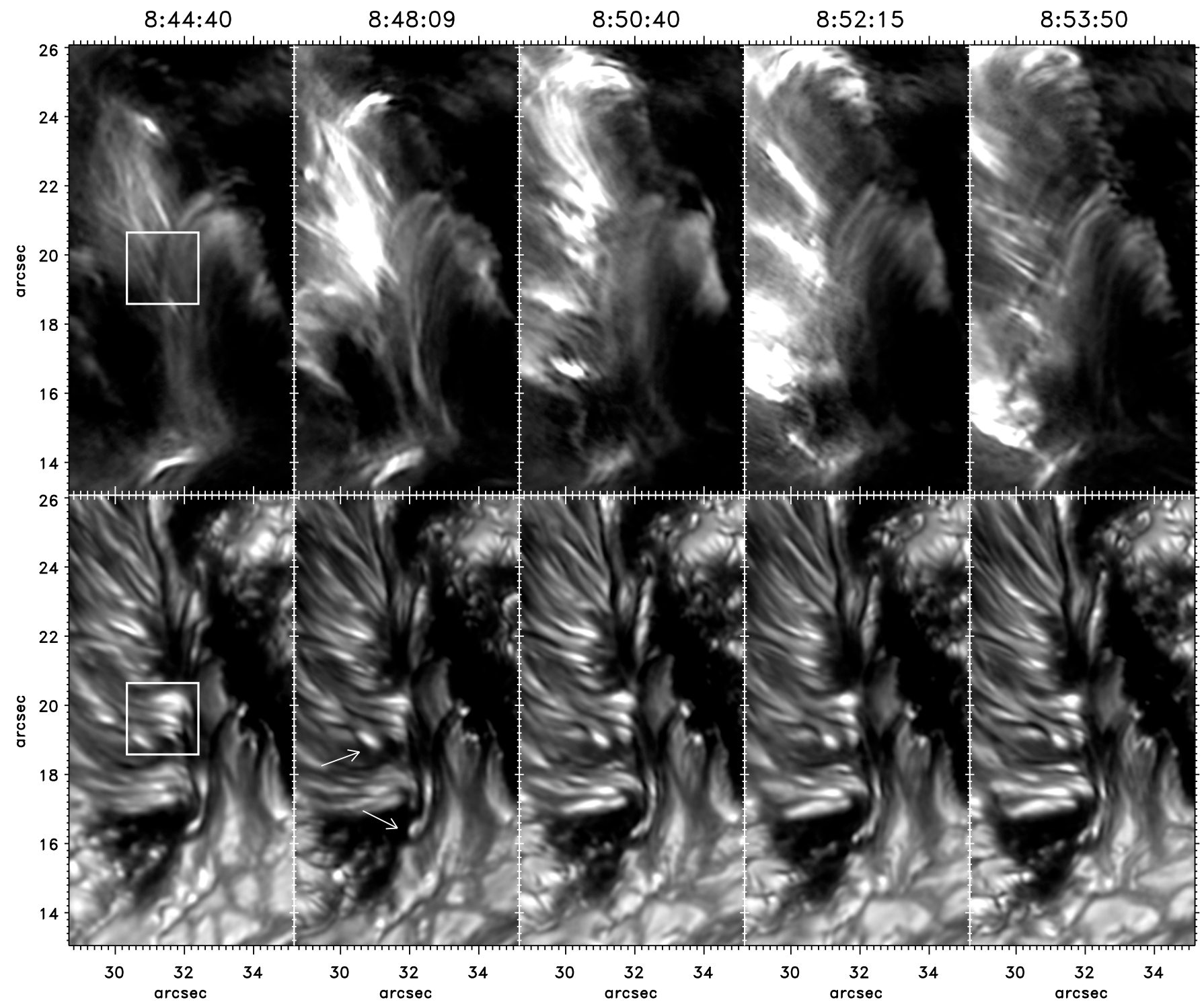

Fig. 14. The onset of the flare in FR2 as seen in the Ca II $H$ line-core (upper panels) and in the blue continuum (lower panels). The white boxes mark the subregion displayed in Fig. 21. Time labels are given in UT.

branches seem to intersect. The longest one (P2) reaches from the upper border of the details displayed in Fig. 17 to their lower border. It seems to submerge below P1 slightly above the small roundish umbra U3 and re-emerges below this umbra. This submergence of P2 below P1 might be also assumed from animating a time series of the blue continuum images. The proper motions therein in both parts of branch $\mathrm{P} 2$ are seen to be directed outward, whereas in the branch P1 the penumbral substructures seem to move inward, i.e. towards DR2, on one side and outward (towards DR1) on its other side. Figure 8 shows a typical structure moving towards DR2. The magnetic field lines in the region below U3 (lower part of P2) must have a loop-like structure since $B_{\mathrm{h}}$ is continuously directed downward and $B_{z}$ is positive close to the $\mathrm{U} 3$ and turns to negative values at a distance of a few arcseconds from $\mathrm{U} 3$.

During the observation sequence, the magnetic structure of this region displays some significant changes. The explanation for these changes that we prefer is that the regions of negative $B_{z}$ are emerging from below, which is mainly reflected by a growth of the regions of negative $B_{z}$, and that this field emergence triggers the flare. In spite of these changes, the intersection of penumbral branches seems to persist during the entire observation period.

\subsection{Dynamic features}

A wide variety of dynamic features is visible on extremely small (spatial and temporal) scales in our data. In Fig. 20 (left panel) the region already shown in Fig. 14 is overplotted with a horizontal flow field obtained from the Ca II $H$ images between 8:45:56 UT and 8:48:47 UT. Before the flare appears, the region shows two interlaced branches of Ca II $H$ fibrils (see upper left panel in Fig. 14). The flow field was derived using LCT with a Gaussian window function of 0.9 width. Within this time interval, a bright cloud is moving along the branch of $\mathrm{Ca}$ II $H$ fibrils that lies above the island of negative $B_{z}$ shown in Fig. 15. This cloud achieves maximum speeds of $25.5 \mathrm{~km} \mathrm{~s}^{-1}$ just as it crosses the island of negative $B_{z}$ (at $\left[32^{\prime \prime}, 18^{\prime \prime}\right]$ in Fig 20). This velocity lies at the upper limit of chromospheric flows typically observed in $\mathrm{H} \alpha$ (see e.g. Al et al. 2004; De Pontieu et al. 2007) or in Ca II 8662 A (see Langangen et al. 2008), although van Noort \& Rouppe van der Voort (2006) found blobs moving with speeds up 

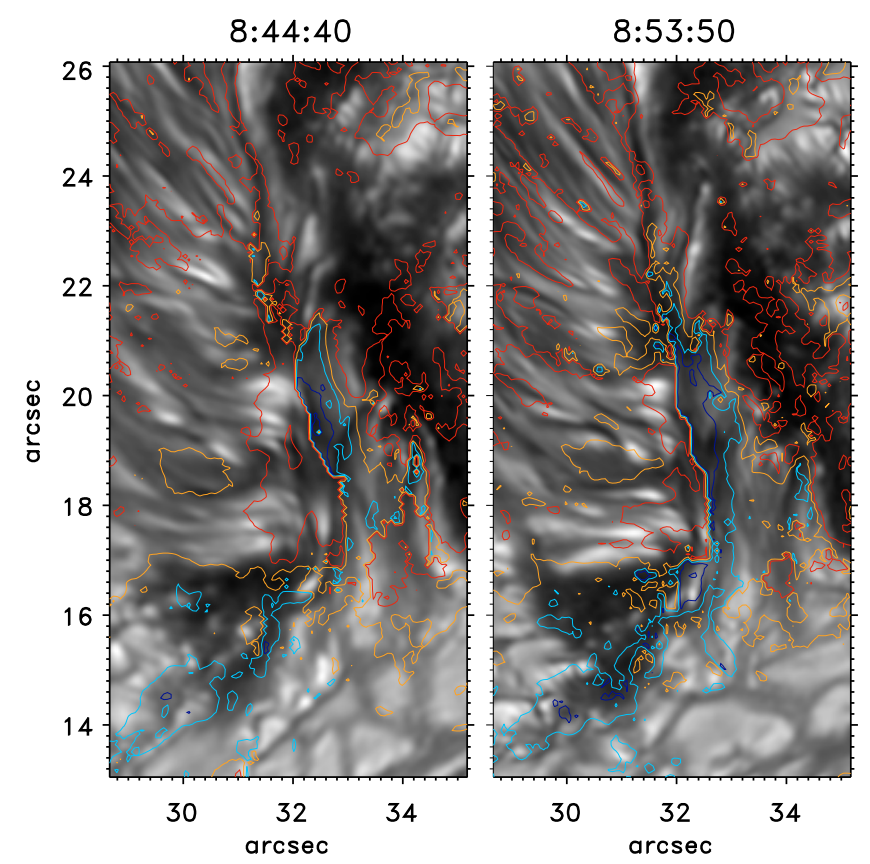

Fig. 15. Vertical magnetic field strength in the region of FR2 before and after the onset of the flare. Contour levels are at $-750 \mathrm{G}$ (blue), $-250 \mathrm{G}$ (green), $250 \mathrm{G}$ (yellow), and $750 \mathrm{G}$ (red). Background images are Gcontinuum data obtained at the times labelled above the panels.

to $240 \mathrm{~km} \mathrm{~s}^{-1}$. Line-of-sight flows exceeding this value are also regularly seen in He I $10830 \AA$ spectra (cf. e.g. Aznar Cuadrado et al. 2005; Lagg et al. 2007; Sasso et al. 2009). The flare eruption takes place (first enhancement of $\mathrm{Ca}$ II $H$ brightness in this region) approximately one minute after the cloud has passed the crossing of the two branches of fibrils and approximately $40 \mathrm{~s}$ after the cloud reaches the upper end of the branch.

During the same period (i.e. approximately between 8:40 UT and 8:47 UT) in the photosphere below, penumbral filaments with typical dark cores (see Scharmer et al. 2002) are located (see Fig. 21). In the uppermost filament visible in the details shown in Fig. 21, a dark roundish structure moves from the dark core of the filament towards the magnetic neutral line (see panels from 8:40:15 UT to 8:43:06 UT in Fig. 21). Its size is only about 0 "' 15 . Later on (see panels from 8:43:06 UT to $8: 46: 35$ UT in Fig. 21), superposed to the lowermost of the three filaments, another tiny but elongated dark structure crosses. This dark structure is about 0 .'15 wide and not longer than 0.3 . In an animation of this subregion, these features appear as conspicuous dark structures, leaving the filaments unmodified. Also the corresponding Doppler maps show no anomalies. The regions close to the centres of the bright heads of the penumbral filaments exhibit strong blueshifts and the dark lanes between the filaments indicate strong redshifts that can be attributed to Evershed flows. These features are also visible in the Ca II $H$ line-wing images. Therefore, they might be ascribed to density enhancements appearing in the temperature minimum or even in the lower chromosphere. several fibrils are visible also in the $\mathrm{Ca}$ II $H$ line-core images (see Fig. 14 and the lower panels of Fig. 21). During the appearance of the dark photospheric structures, these fibrils are already increasing their intensity (onset of the flare). In the contrast-enhanced Ca II $H$ image from 8:46:15 UT in Fig. 21, the fast-moving cloud described in the previous paragraph can also be seen. It is located in the lower right corner of this panel above two conspicuous bright fibrils and just crosses the magnetic neutral line.
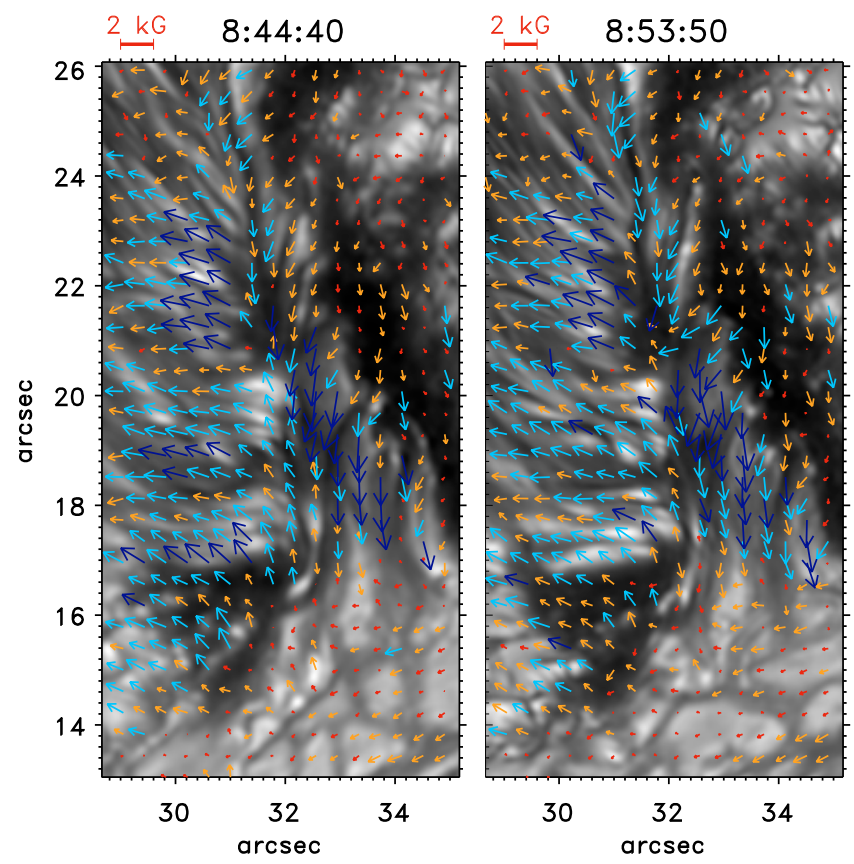

Fig. 16. Direction and strength of the horizontal magnetic field in the region of FR2 before and after the onset of the flare. Arrow colours denote field strengths $B_{\mathrm{h}}<500 \mathrm{G}$ (red), $500 \mathrm{G} \leq B_{\mathrm{h}}<1000 \mathrm{G}$ (yellow), $1000 \mathrm{G} \leq B_{\mathrm{h}}<1500 \mathrm{G}$ (green), and $B_{\mathrm{h}} \geq 1500 \mathrm{G}$ (blue). Background images are G-continuum data obtained at the times labelled above the panels.

The uppermost of the three penumbral filaments displayed in Fig. 21 shows another dynamic feature that appears as a bright extension of this filament (Fig. 21, panel from 8:46:35 UT). This bright extension is (considering that the spatial resolution of the magnetogram is not better than $0 .{ }^{\prime \prime} 2-0.3$ ) located exactly below the magnetic neutral line that separates the island of negative $B_{z}$ from the penumbral filaments where $B_{z}>0$. This structure is a remnant of a slowly decaying bright penumbral filament located along the magnetic neutral line approximately $25 \mathrm{~min}$ prior to the flare eruption. It disappears completely during the onset of the flare. The less conspicuous structure marked in Fig. 21 is another remnant of the same penumbral filament.

The two previously discussed features show clearly that the photosphere below the flare undergoes a significant restructuring shortly before the flare erupts, although only on extremely small scales.

Another striking feature that can be seen in Fig. 21 is the strongly different orientation of photospheric penumbral fibrils and chromospheric Ca II $H$ fibrils. The two types of features are misaligned by an angle of more than $45^{\circ}$. Such a misalignment of photospheric and chromospheric structures can be seen in several parts of this region. The region around $\left[26^{\prime \prime}, 26^{\prime \prime}\right]$ in the second panel (8:48:09 UT) of Fig. 17 shows another example. There the misalignment is even of the order of $75^{\circ}$. The photospheric field is roughly aligned with the penumbral fibrils (compare left panel of Fig. 9 with the upper left panel of Fig. 3). If we now assume that the Ca II $H$ fibrils mark chromospheric field lines, then it would appear that the field structure in the observed region not only shows strong horizontal shear but also a strong vertical rotation; i.e., the flare occurs in a region of strong helicity. The flare does not fully remove the misalignment of chromospheric and photospheric structures. As can be seen in the upper right panels of Figs. 14 and 17, intersecting branches of Ca II $H$ fibrils remain in the analysed region and the underlying penumbral 


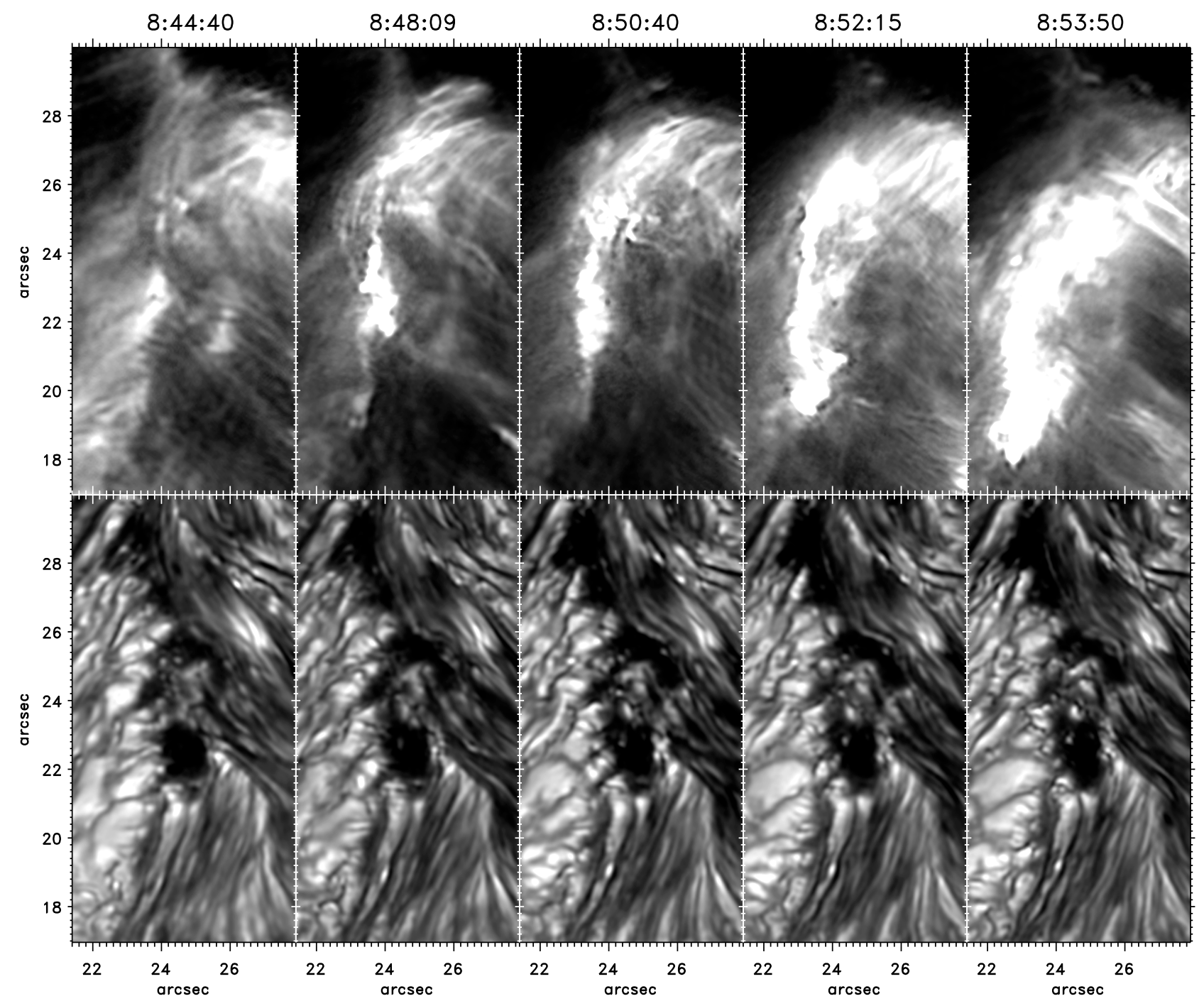

Fig. 17. The onset of flare ribbon FR1 as seen in the Ca II $H$ line-core (upper panels) and in the blue continuum (lower panels). Time labels are given in UT.

fibrils remain misaligned with at least one branch of the overlying Ca II $H$ fibrils. Different orientations in the photosphere and chromosphere may also have to do with different inclinations of photospheric and chromospheric structures with respect to the solar surface, since the sunspot was significantly far removed from disk centre $\left(\theta=40.15^{\circ}\right)$ at the time of observation.

Another dynamical feature, visible during the main phase of the flare, is shown in the right panel of Fig. 20. This figure shows the $\mathrm{Ca}$ II $H$ line-wing intensity in the region between the two flare ribbons. Overplotted is the horizontal flow field obtained from the Ca II $H$ line-wing images between 8:50:40 UT and 8:56:41 UT. In the centre of the cutout (at [30".5, 27"]; see also Fig. 12, panel from 8:52:52 UT), a bright front is moving towards the top-right. The velocities (using LCT with a 0.9 window function) achieve values up to $4.8 \mathrm{~km} \mathrm{~s}^{-1}$. It is co-spatial with the outer border of the rapidly expanding flare loop visible in the Ca II $H$ line-centre images (see Fig. 14) and is, thus, not exactly co-spatial with the brightest parts of the line-core images. The width of this front is only approximately 0.25 . If this bright front were only caused by the contribution of the linecentre intensities to the line-wing images (since the filter width is larger than the wavelength difference between the two channels), it should be co-spatial with the brightest parts of the flaring region. Although the detector was saturated in some regions, this is definitely not the case. We, therefore, assume a strong blueshift of the CaII $H$ line in the front and also in the fibrils flashing in the line-wing images. Using the wavelength difference of $0.6 \AA$ between the two channels results in a speed of $45 \mathrm{~km} \mathrm{~s}^{-1}$.

A second region of fast horizontal motions is located in the lower left corner of the right panel of Fig. 20. These motions are related to fibrils appearing bright in the $\mathrm{Ca}$ II $H$ line-wing. Since a distinct moving wavefront is not visible, it remains unclear if the bright fibrils are really moving or whether we only see various fibrils flashing at different times that mislead the LCT algorithm.

\section{Discussions and conclusions}

The observed disturbed penumbral region of the trailing spot of the bipolar active region NOAA 10904 shows an extremely complex magnetic field and flow configuration in the photosphere and a hardly less complex structure of $\mathrm{Ca}$ II $H$ fibrils, which represent the lower chromosphere. Summarising the deductions made in the previous sections, in Fig. 22 the atmospheric structure in the regions of the two flare ribbons is sketched. One flare 


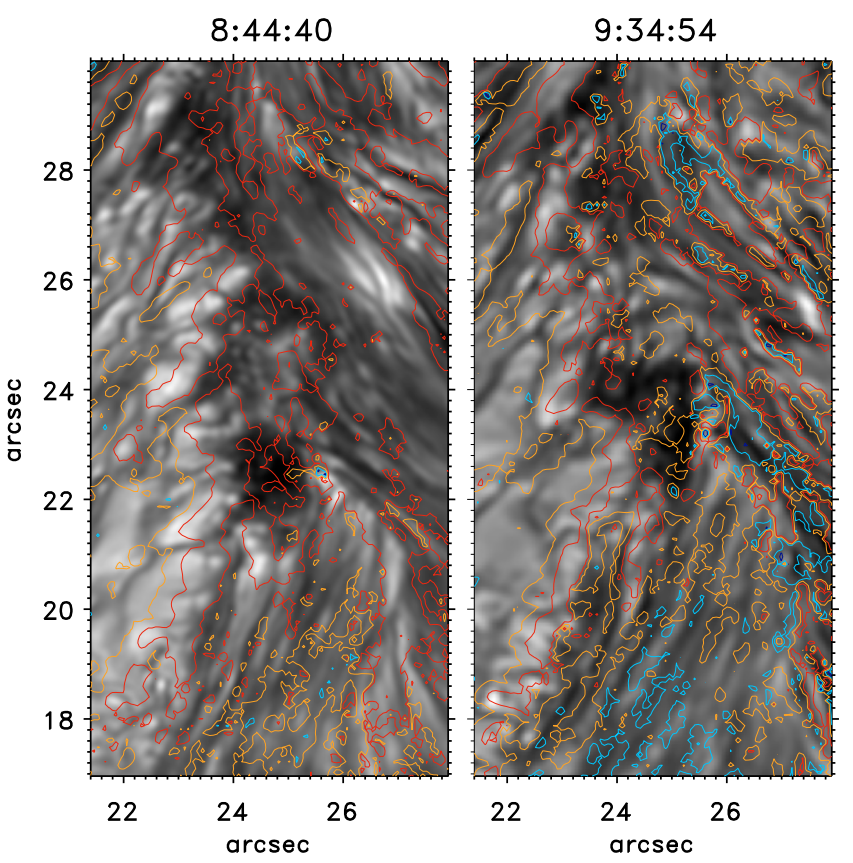

Fig. 18. Vertical magnetic field strength around FR1 at the onset and $40 \mathrm{~min}$ after the flare. Contour levels are at $-750 \mathrm{G}$ (blue), $-250 \mathrm{G}$ (green), $250 \mathrm{G}$ (yellow), and $750 \mathrm{G}$ (red). Background images are G-continuum data obtained at the times labelled above the panels.
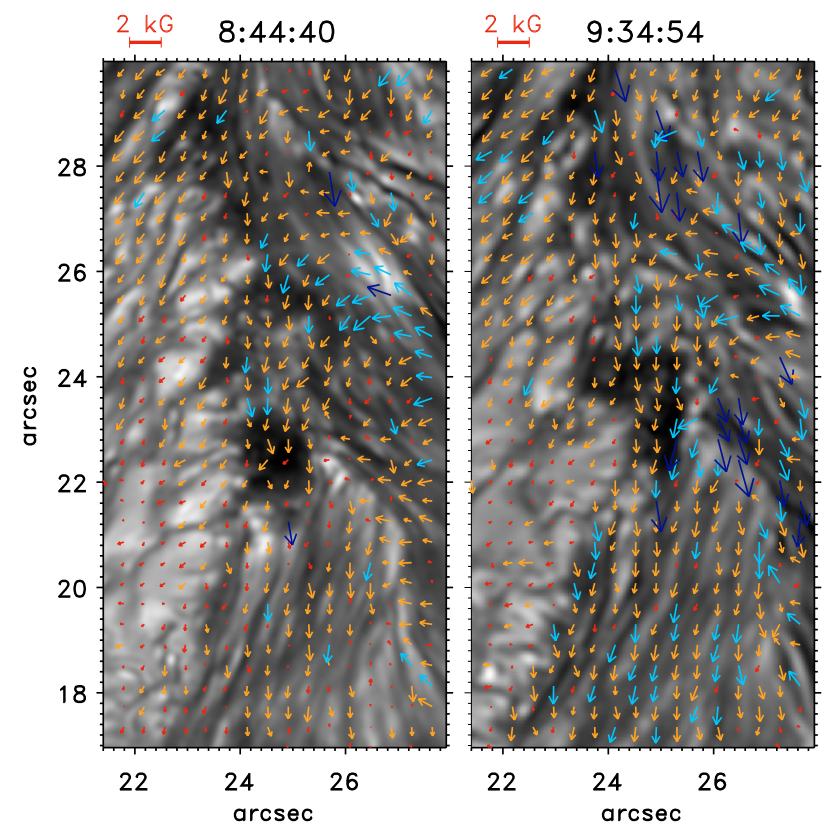

Fig. 19. Direction and strength of the horizontal magnetic field around FR 1 at the onset and $40 \mathrm{~min}$ after the flare. Arrow colours denote field strengths $B_{\mathrm{h}}<500 \mathrm{G}$ (red), $500 \mathrm{G} \leq B_{\mathrm{h}}<1000 \mathrm{G}$ (yellow), $1000 \mathrm{G} \leq$ $B_{\mathrm{h}}<1500 \mathrm{G}$ (green), and $B_{\mathrm{h}} \geq 1500 \mathrm{G}$ (blue). Background images are G-continuum data obtained at the times labelled above the panels.

ribbon (FR1) is located close to the small umbra U3, roughly parallel to the penumbral branch $\mathrm{P} 2$. The other flare ribbon (FR2) is located above the endpoints of penumbral branches P1 and $\mathrm{P} 3$ where they meet $\mathrm{P} 4$.

Flare ribbon FR2 (cf. Fig. 14) includes an island of inverse $B_{z}$, which seems to contain the footpoints of loop-like magnetic fields. In close proximity to this island, a branch of penumbral filaments (P1) with a polarity of the magnetic field and the
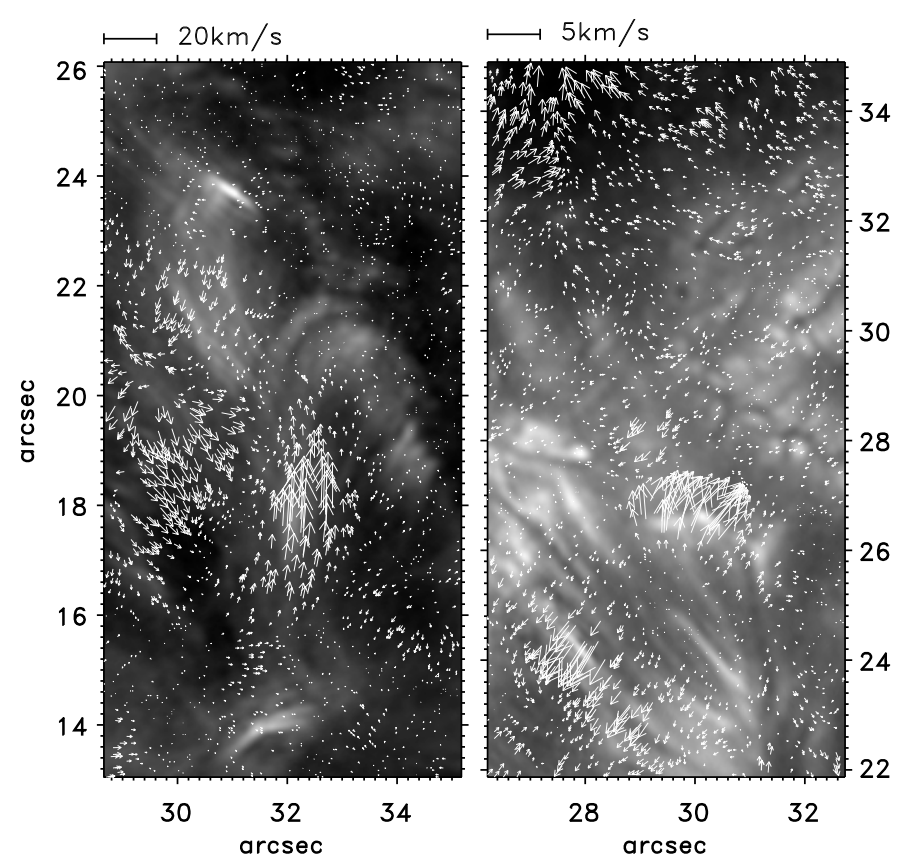

Fig. 20. Left panel: horizontal flow field as obtained from the Ca II $H$ line-core images between 8:45:56 UT and 8:48:47 UT. The cutout displays the same region as shown in Figs. 14-16. Right panel: horizontal flow field as obtained from the Ca II $H$ line-wing images between 8:50:40 UT and 8:56:41 UT in the region marked by a white box in Fig. 12.

Evershed flow, which is opposite to the main polarity of the spot penumbra, emerges from the subsurface layers.

The region around flare ribbon FR1 (cf. Fig. 17) shows several crossing branches of penumbral filaments, particularly at the endpoints (DR2 and DR3, see Fig. 9) of P1 and P3, which carry the oppositely directed Evershed motions and in which the magnetic fields are oppositely directed to the main penumbral field direction.

Both regions are overlaid by several crossing branches of fibrils visible in $\mathrm{Ca}$ II $H$. Some of these branches show almost no motions, only strong brightness enhancements during the flare, and others show rapid expansion. Some of the $\mathrm{Ca}$ II $H$ fibrils are found to modify their azimuthal direction during the flare, such that a fibril that starts strongly skewed relative to the underlying photospheric penumbral fibril ends up being more nearly parallel.

The obtained structure can be fitted into a global picture of the flare eruption as developed in Falconer et al. (2000). The observed region includes several islands of negative magnetic polarity in the positive polarity region of NOAA 10904. According to Falconer et al. (2000), the islands of inverse polarity are connected to the adjacent regions by short loops leading to a closed core field enveloping the neutral line around the islands (see their Fig. 3). Close to the magnetic neutral lines, the fields are strongly sheared, which is very often the case prior to a flare eruption (Hagyard et al. 1984). A separatrix surface encloses the islands. The footpoints of the separatrix surface enclose the entire region. In our case, this intersection of the separatrix with the surface marks the footpoints of the branches of Ca II $H$ fibrils, which are connected to the interior of the disturbed region and can be easily identified by a continuous brightness enhancement prior to and after the flare eruption.

Since the pre-flare structure of both the chromosphere and the photosphere of the observed region does not differ much 

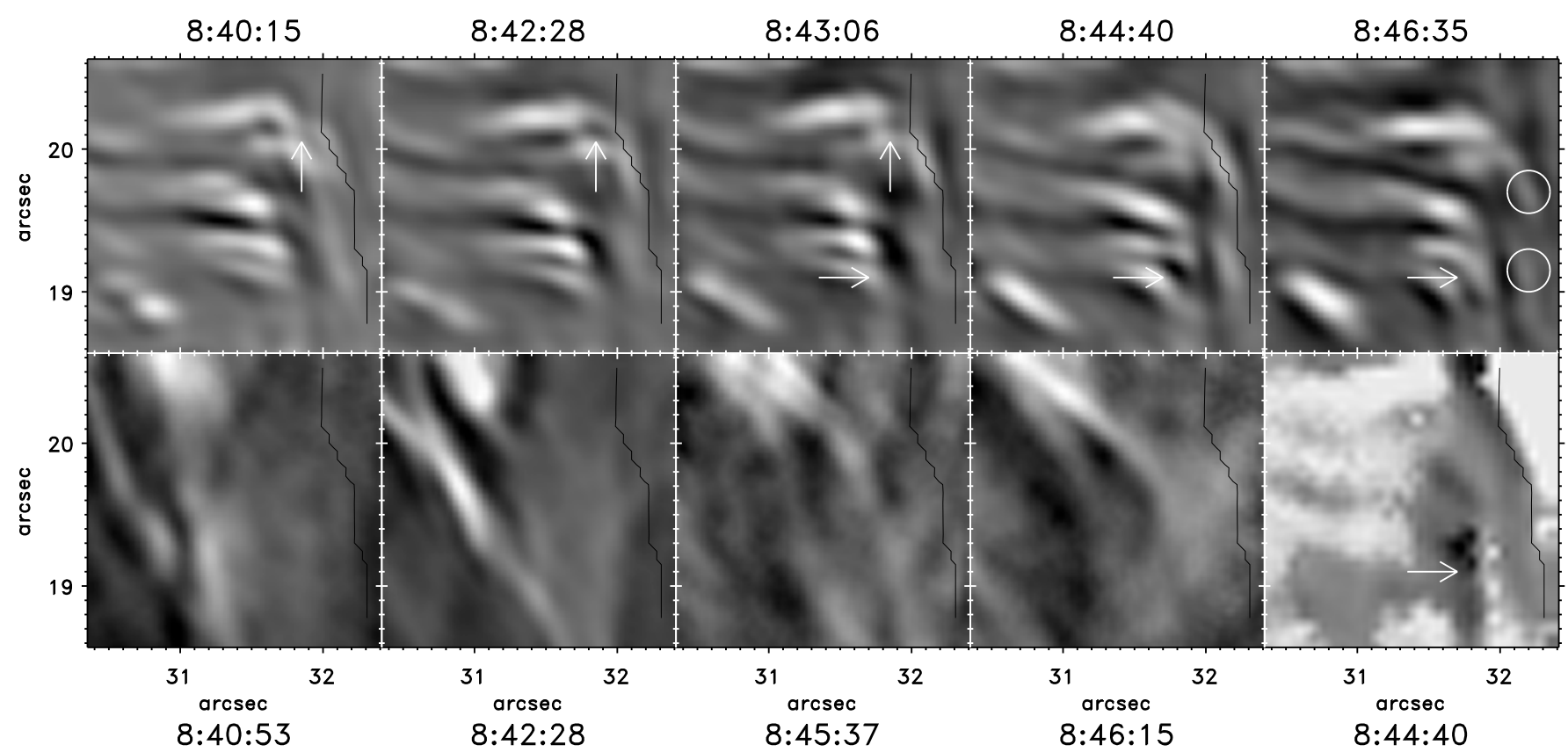

Fig. 21. Evolution of penumbral branch $P 1$ at the onset of the flare (FR2). Upper panels: contrast-enhanced G-continuum images. Dark structures are crossing the dark-cored penumbral filaments (arrows define reference positions). Bright remnants of a decaying penumbral filament are marked by circles. Lower panels: contrast-enhanced Ca II $H$ line-core images and and the corresponding Doppler map (lower right panel) scaled to values between $v_{\mathrm{LOS}}=-1.5 \mathrm{~km} \mathrm{~s}^{-1}$ (white) and $v_{\mathrm{LOS}}=1 \mathrm{~km} \mathrm{~s}^{-1}$ (black). The overplotted line shows the magnetic neutral line $\left(B_{z}=0\right)$ at 8:44:40 UT. The location of this subregion is marked with white boxes in Figs. 1 and 14. Time labels are given in UT.

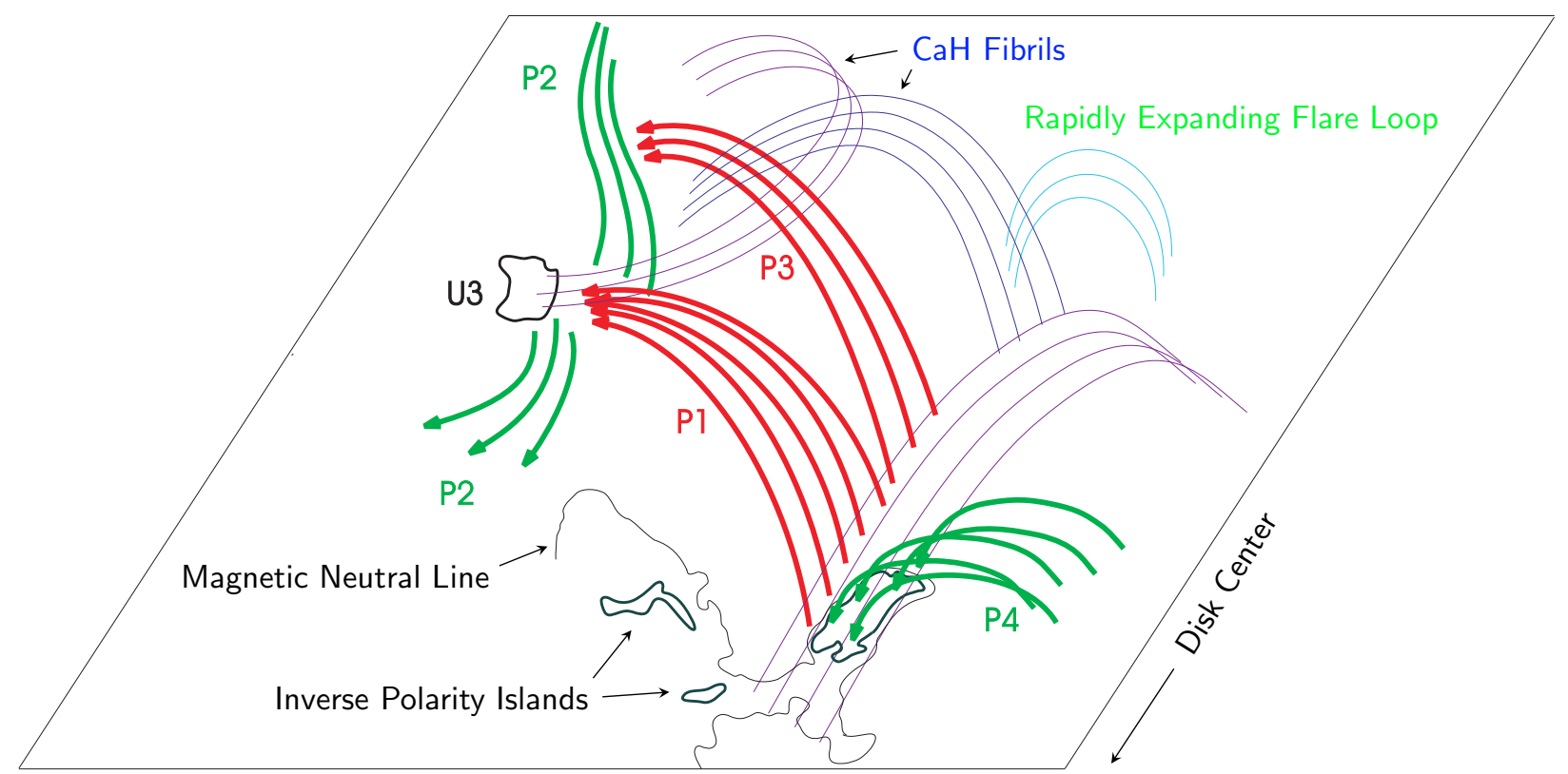

Fig. 22. Schematic drawing of the disturbed penumbral region during the onset of the flare. Thick lines denote photospheric penumbral fibrils (colours of the different branches are identical with those in Fig. 2; arrows delineate the magnetic field direction); thin coloured lines denote chromospheric Ca II $H$ fibrils; black contours mark the magnetic neutral line (thin) and the small umbra U3 (thick). The islands of inverse $B_{z}$ are displayed as thick dark-green contours. The flare onset regions are located close to intersection of P1 and P2 (FR1) and at the connection area of P1 and P4 (FR2).

from the post-flare structure and because the observed eruption is only a weak flare (GOES magnitude B7.8) the global topology of the region does not change during the flare. However, the high resolution of the analysed data made it possible to study smallscale restructuring of the atmosphere during a weak flare for the first time. Primarily, we found a sizable increase in the islands of inverse polarity. This is consistent with the long-term evolution of the spot, which can be traced, e.g., on SOHO/MDI continuum images (see Fig. 23). The formation of the sunspot is not completely clear, since it appeared for the first time on August 10, 2006 on the extreme eastern limb of the Sun, but it seems that it was formed by a merger of two spots of the same polarity. The two spots (represented by the two large umbrae visible in Fig. 1) achieved closest proximity around August 12, 2006 and started to re-split (and decay) in the following days until they were completely separated on August 16, 2006. The observed 


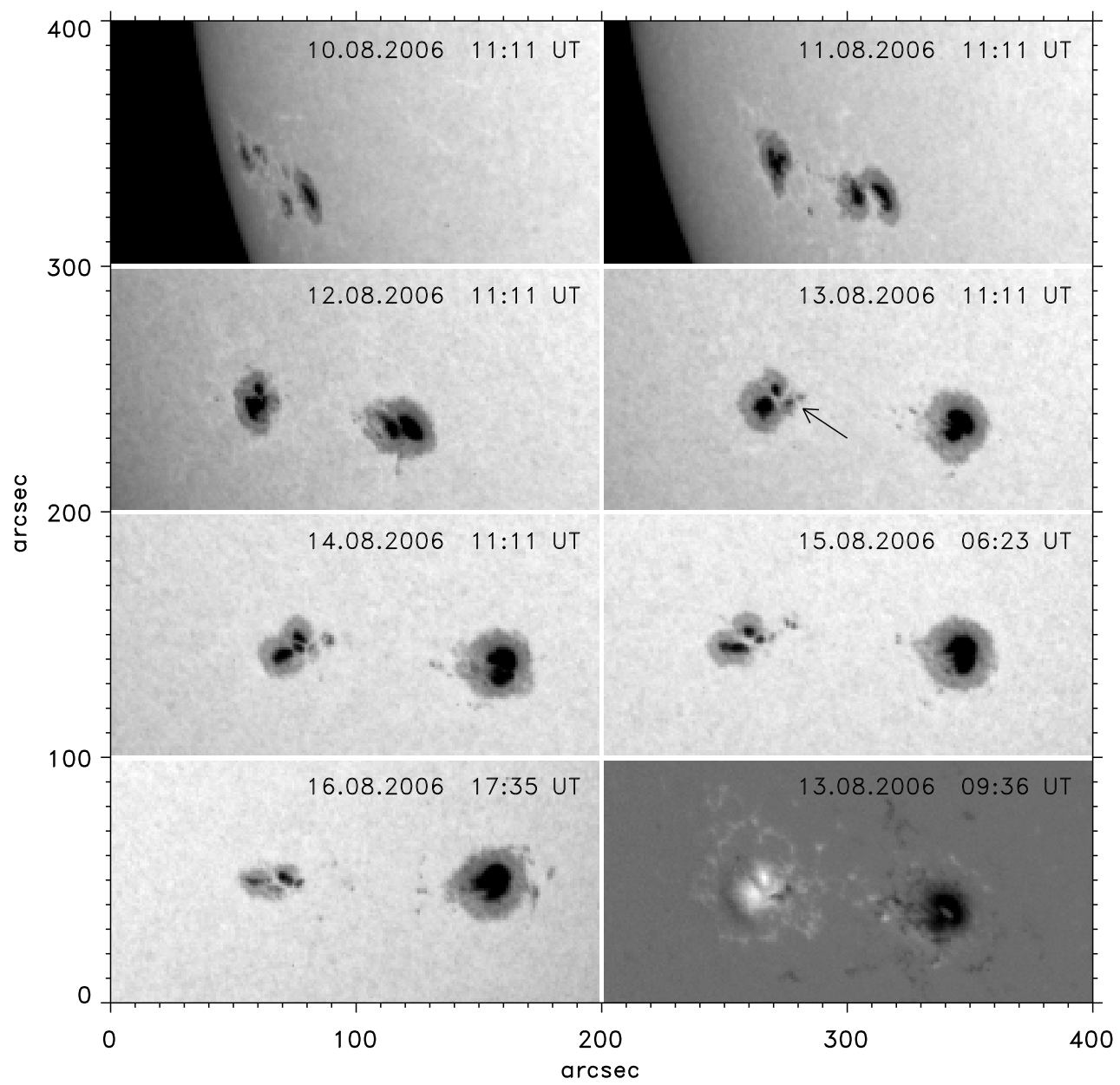

Fig. 23. Evolution of active region NOAA 10904 as observed in white light by SOHO/MDI. The arrow point to the flare region and the lower right panel shows an MDI magnetic field map from August 13, 2006. The formation of the trailing sunspot remains unclear, but it decayed rapidly after August 13, 2006.

crossing of penumbral branches of opposite polarity might stem from the penetration of the penumbra of one spot into that of the other one during the merger of the two spots. This penetration might also drive the emergence of reversed polarity magnetic flux during the flare eruption. The eruption even might be triggered by this flux emergence. This interpretation is supported by several B-class events in the considered active region having been registered by the GOES/SXI instrument within several hours before and after the one discussed here. However, this one with magnitude B7.8 was by far the strongest one. The model of a restructuring of the intersecting penumbral magnetic fields is, furthermore, supported by a single penumbral filament aligned with the magnetic neutral line of one of the inverse-polarity islands (see Fig. 21) slowly decaying from about $20 \mathrm{~min}$ prior to the flare eruption on. Its last visible remnants dissappear simultaneously with the onset of the flare.

Based on these results the actual triggering mechanism must also be searched in the dynamics of the regions of the intersecting penumbrae. However, the pre-flare dynamics of these regions only show a few conspicuous features. One of these features is a fast-moving cloud visible in the Ca II $H$ line-core images (see Fig. 20). This cloud crosses the point of the flare onset (an intersection of two branches of Ca II $H$ fibrils) approximately one minute before the onset of the flare. The trajectory of this cloud motion is co-spatial with the decaying penumbral filament located at the neutral line of the magnetic island. We also find a strongly sheared magnetic field configuration along this neutral line. This conjuncture of events suggests a close connection with the flare onset.

Other features are tiny dark structures crossing the bright heads of dark-cored penumbral filaments. These hitherto unknown phenomena are also located close to the previously mentioned magnetic neutral line below the crossing of two branches of Ca II $H$ fibrils. While the event takes place, the fibrils are already starting to increase their intensity at a distance of about one arcsecond from the event. The dark structures leave the penumbral filaments unmodified. Therefore, we assume that they are some kind of dense matter crossing the filaments in the upper photosphere. They are also visible, although much less conspicuous, in the $\mathrm{Ca}$ II $H$ line-wing images. It is hard to decide whether these structures are related to the triggering mechanism of the flare. More likely, they are an early manifestation of the flare eruption.

The entire analysed disturbed penumbral region is connected with fast motions. Most strikingly, we found strong photospheric Doppler shifts that correspond to velocities of up to $7 \mathrm{~km} \mathrm{~s}^{-1}$. These motions are most likely Evershed flows which achieve their maximum speeds close to the onset points of the flare eruption. At these points, convergence centres of the horizontal flows have also been detected and the magnetic fields show conspicuous discontinuities. Therefore, we speculate that the intersection of penumbrae may lead to a contraction of their interlaced branches at certain points which accelerates the Evershed flows. Several penumbral grains located in close proximity to the 


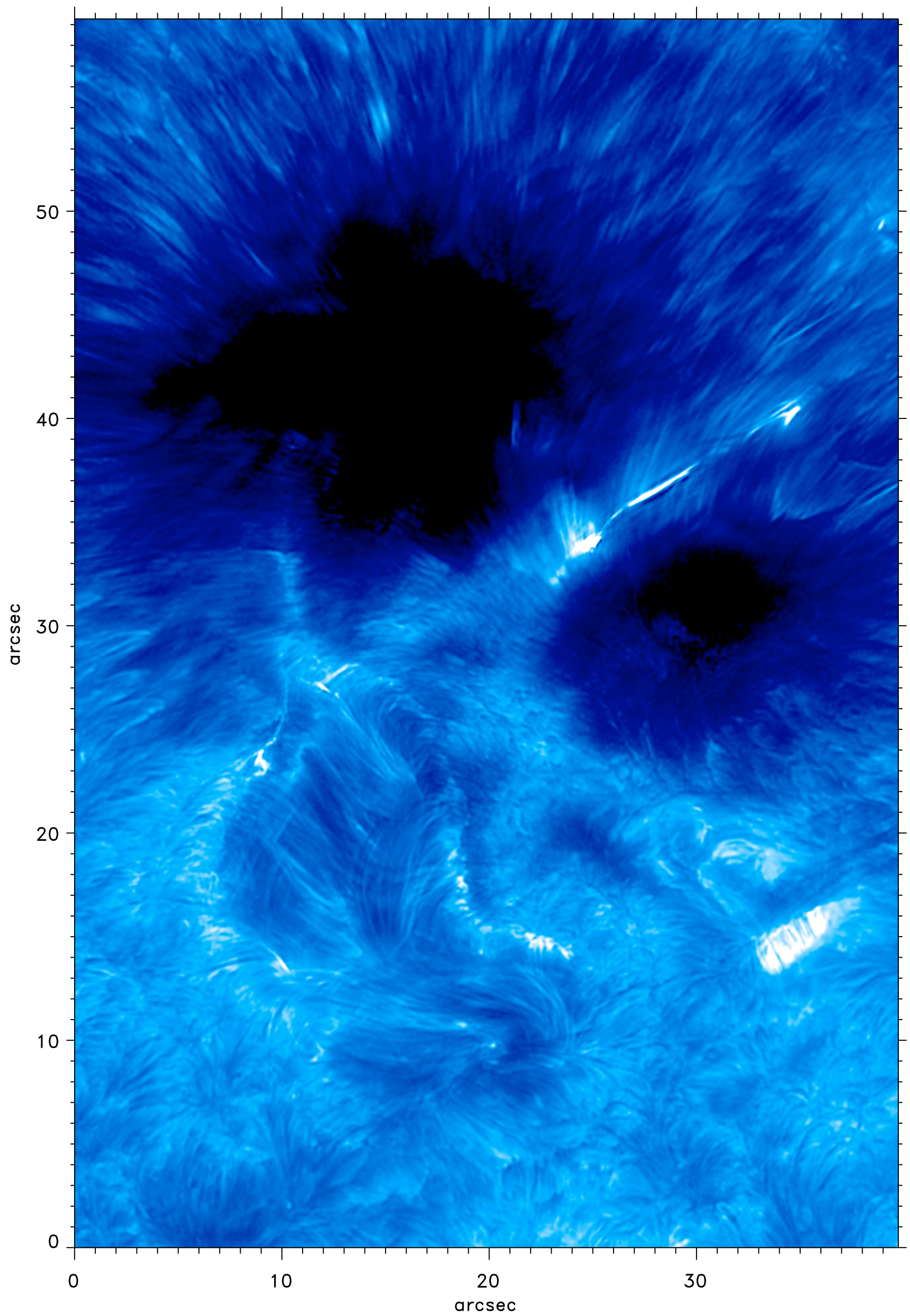

Fig. 24. Full field of viw of the Ca II $H$ observations. The image shows the sunspot and the flaring region at 9:58 UT which is about one hour after the flare eruption. 
convergence centres (see e.g. Fig. 8) also move with unusually high speeds, which may or may not support our hypothesis. Fast and even supersonic flows in confined penumbral regions close to the magnetic neutral lines in $\delta$-spots were already found by Martínez Pillet et al. (1994) and Lites et al. (2002), while evidence of supersonic Evershed flow in normal penumbrae has been provided by Borrero et al. (2005) and Bellot Rubio et al. (2007).

Fast motions were also detected in the chromosphere and upper photosphere. Apart from a fast moving cloud visible in the Ca II $H$ line-core images (see above), we found several bright features flashing up in the $\mathrm{Ca}$ II $H$ line-wing images. The simplest explanation for these flashes is a strong blueshift of the $\mathrm{Ca}$ II $H$ line in these regions. The line-wing images were obtained approximately $0.6 \AA$ out of the line centre. This value corresponds to blueshifts of $45 \mathrm{~km} \mathrm{~s}^{-1}$. We may, therefore, assume velocities of several $10^{4} \mathrm{~m} \mathrm{~s}^{-1}$. Beside these flashes, the only feature visible in the $\mathrm{Ca}$ II $H$ line-wing and related to the flare, which lasts longer than a few images, is the outgoing brightness front of a rapidly expanding branch of loop-like Ca II $H$ fibrils. The front, visible in the line-wing, might be assumed to correspond to the footpoints of the loop-like structures. There the chromospheric magnetic field lines should be mainly vertical which is consistent with fast upflows.

Acknowledgements. The authors are grateful to an anonymous referee for helpful comments. Part of this work was supported by the WCU grant (No. R31-10016) from the Korean ministry of Education, Science and Technology. The Swedish Solar Telescope (SST) is operated on the island of La Palma by the Institute for Solar Physics of the Royal Swedish Academy of Sciences in the Spanish Observatorio del Roque de los Muchachos of the Instituto de Astrifísica de Canarias. We thank the telescope stuff, Helena Uthas, Rolf Kever and Michiel van Noort, for their kind support with the SST.

\section{References}

Al, N., Bendlin, C., Hirzberger, J., Kneer, F., \& Trujillo Bueno, J. 2004, A\&A, 418, 1131

Aschwanden, M. J., Winebarger, A., Tsiklauri, D., \& Peter, H. 2007, ApJ, 659, 1653

Aznar Cuadrado, R., Solanki, S. K., \& Lagg, A. 2005, in Chromospheric and Coronal Magnetic fields, ed. D. E. Innes, A. Lagg, \& S. K. Solanki, ESA SP-596, 49.1

Bellot Rubio, L. R., Tsuneta, S., Ichimoto, K., et al. 2007, ApJ, 668, L91

Benz, A. O. 2008, LRSP, 5, 1B

Borrero, J. M., Lagg, A., Solanki, S. K., \& Collados, M. 2005, A\&A, 436, 333

Bovelet, B., \& Wiehr, E. 2003, A\&A, 412, 249
Cabrera Solana, D., Bellot Rubio, L. R., Beck, C., \& del Toro Iniesta, J. C. 2007, A\&A, 475, 1067

de Boer, C. R. 1996, A\&AS, 120, 195

De Pontieu, B., Hansteen, V. H., Rouppe van der Voort, L., van Noort, M., \& Carlsson, M. 2007, ApJ, 655, 624

Falconer, D. A., Gary, G. A., Moore, L., \& Porter, J. G. 2000, ApJ, 528, 1004

Hagyard, M. J., Smith, J. B., Jr., Teuber, D., \& West, A. 1984, Sol. Phys., 91, 115

Ichimoto, K., Suematsu, Y., Tsuneta, S., et al. 2007, Science, 318, 159

Krieg, J., Wunnenberg, M., Kneer, F., Koschinsky, M., \& Ritter, C. 1999, A\&A, 343, 983

Krucker, S. 2002, AdSpR, 30, 493

Künzel, H. 1960, AN, 285, 271

Lagg, A., Woch, J., Krupp, N., \& Solanki, S. K. 2004, A\&A, 414, 1109

Lagg, A., Woch, J., Solanki, S. K., \& Krupp, N. 2007, A\&A, 462, 1147

Langangen, Ø., Carlsson, M., Rouppe van der Voort, L., Hansteen, V., \& De Pontieu, P. 2008, ApJ, 673, 1194

Li, H., Sakurai, T., Ichimoto, K., \& UeNo, S. 2000a, PASJ, 52, 465

Li, H., Sakurai, T., Ichimoto, K., \& UeNo, S. 2000b, PASJ, 52, 483

Lin, R. P., Schwartz, R. A., Kane, S. R., Pelling, R. M., \& Hurley, K. C. 1984, ApJ, 283, 421

Lites, B. W., Low, B. C., Martínez Pillet, V., et al. 1995, ApJ, 446, 877

Lites, B. W., Socas-Navarro, H., Skumanich, A., \& Shimizu. T. 2002, ApJ, 575, 1131

Metcalf, T. R., Leka, K. D., Barnes, G., et al. 2006, Sol. Phys., 237, 267

Martínez Pillet, V., Lites, B. W., Skumanich, A., \& Degenhardt, D. 1994, ApJ, 425, L113

November, L. J., \& Simon, G. W. 1988, ApJ, 333, 427

Orozco Suarez, D., Bellot Rubio, L. R., del Toro Iniesta, J. C., et al. 2007, PASJ, $59, \mathrm{~S} 837$

Pauluhn, A., \& Solanki, S. K. 2007, A\&A, 462, 311

Pehlemann, E., \& von der Lühe, O. 1989, A\&A, 216, 337

Pietarila, A., Hirzberger, J., Zakharov, V., \& Solanki, S. K. 2009, A\&A, submitted

Rempel, M., Schüssler, M., \& Knölker, M. 2009, ApJ, 691, 640

Rutten, R. J. 2007, in The Physics of Chromospheric Plasmas, ed. P. Heinzel, I. Dorotovič, \& R. J. Rutten, ASP Conf. Ser., 368, 27

Sasso, C., Lagg, A., \& Solanki, S. K. 2009, in prep.

Scharmer, G. B., Gudiksen, B. V., Kiselman, D., Löfdahl, M. G., \& Rouppe van der Voort, L. H. M. 2002, Nature, 420, 151

Scharmer, G. B., Bjelksjö, K., Korhonen, T. K., Lindberg, B., \& Petterson, P. 2003, in Innovative Telescopes and Instrumentation for Solar Astrophysics, ed. S. L. Keil, \& S. V. Avakjan, Proc. SPIE, 4853, 341

Schlichenmaier, R., Jahn, K., \& Schmidt, H. U. 1998, A\&A, 337, 897

Schmieder, B., Hagyard, M. J., Ai, G., et al. 1994, Sol. Phys., 150, 199

Selbing, J. 2005, Master thesis, Stockholm Observatory

Sobotka, M., \& Sütterlin, P. 2001, A\&A, 380, 714

Su, Y., Golub, L., van Ballegooijen, A., et al. 2007, PASJ, 59, S785

Title, A. M., \& Rosenberg, W. J. 1981, Opt. Eng., 20, 815

van Noort, M. J., \& Rouppe van der Voort, L. H. M. 2006, ApJ, 648, L67

Wang, H. 2006, ApJ, 649, 490

Wang, T. J., Innes, D. E., \& Solanki, S. K. 2006, A\&A, 455, 1113

Weigelt, G. P. 1977, Opt. Comm., 21, 55

Zakharov, V., Hirzberger, J., Riethmüller, T., Solanki, S. K., \& Kobel, P. 2008, A\&A, 488, L17 